\title{
Congenital ablation of Tacr 2 reveals overlapping and redundant roles of NK2R signaling in the control of reproductive axis
}

\author{
Encarnacion Torres, ${ }^{1,2}$ Inmaculada Velasco, ${ }^{1,2}$ Delphine Franssen, ${ }^{1,2}$ Violeta Heras, ${ }^{1,2}$ Francisco Gaytan, ${ }^{1,2,3}$ \\ Silvia Leon, ${ }^{1,2,3,4}$ Victor M. Navarro, ${ }^{4}$ Rafael Pineda, ${ }^{1,2,3}$ M. Luz Candenas, ${ }^{5}$ Antonio Romero-Ruiz, ${ }^{1,2}$ and \\ (1) Manuel Tena-Sempere ${ }^{1,2,3,6}$ \\ ${ }^{1}$ Department of Cell Biology, Physiology and Immunology, Instituto Maimonides de Investigación Biomédica de Córdoba \\ (IMIBIC), University of Córdoba, Córdoba, Spain; ${ }^{2}$ Hospital Universitario Reina Sofía, Córdoba, Spain; ${ }^{3}$ CIBER Fisiopatología \\ de la Obesidad y Nutrición (CIBEROBN), Instituto de Salud Carlos III, Córdoba, Spain; ${ }^{4}$ Division of Endocrinology, Department \\ of Medicine, Diabetes, and Hypertension, Brigham and Women's Hospital, Harvard Medical School, Boston, Massachusetts; \\ ${ }^{5}$ Instituto de Investigaciones Químicas, CSIC, Seville, Spain; and ${ }^{6}$ Research Centre for Integrative Physiology and \\ Pharmacology, Institute of Biomedicine and Turku Center for Disease Modeling, University of Turku, Turku, Finland
}

\begin{abstract}
Tachykinin (TAC) signaling is an important element in the central control of reproduction. TAC family is mainly composed of substance $P(S P)$, neurokinin A (NKA), and NKB, which bind preferentially to NK1, NK2, and NK3 receptors, respectively. While most studies have focused on the reproductive functions of NKB/NK3R, and to a lesser extent SP/NK1R, the relevance of NK2R, encoded by Tacr2, remains poorly characterized. Here, we address the physiological roles of NK2R in regulating the reproductive axis by characterizing a novel mouse line with congenital ablation of Tacr2. Activation of NK2R evoked acute luteinizing hormone (LH) responses in control mice, similar to those of agonists of NK1R and NK3R. Despite the absence of NK2R, Tacr2 ${ }^{-1-}$ mice displayed only partially reduced LH responses to an NK2R agonist, which, nonetheless, were abrogated after blockade of NK3R in $T a c r 2^{-1-}$ males. While $T a c r 2^{-1-}$ mice displayed normal pubertal timing, LH pulsatility was partially altered in Tacr2 ${ }^{-/-}$ females in adulthood, with suppression of basal LH levels, but no changes in the number of LH pulses. In addition, trends for increase in breeding intervals were detected in $\mathrm{Tacr} 2^{-1-}$ mice. However, null animals of both sexes were fertile, with no changes in estrous cyclicity or sex preference in social behavioral tests. In conclusion, stimulation of NK2R elicited LH responses in mice, while congenital ablation of Tacr2 partially suppressed basal and stimulated LH secretion, with moderate reproductive impact. Our data support a modest, albeit detectable, role of NK2R in the control of the gonadotropic axis, with partially overlapping and redundant functions with other tachykinin receptors.
\end{abstract}

NEW \& NOTEWORTHY We have explored here the impact of congenital ablation of the gene (Tacr2) encoding the tachykinin receptor, NK2R, in terms of neuroendocrine control of the reproductive axis, using a novel Tacr2 KO mouse line. Our data support a modest, albeit detectable, role of NK2R in the control of the gonadotropic axis, with partially overlapping and redundant functions with other tachykinin receptors.

gonadotropins; neuroendocrinology; neurokinin A; tachykinins; tachykinin receptors

\section{INTRODUCTION}

The mammalian reproductive system, essential for the perpetuation of species, is controlled by a complex network of central and peripheral regulatory inputs, which integrate at the hypothalamic-pituitary-gonadal (HPG) axis. A major hallmark of the functioning of this neuroendocrine axis is the pulsatile secretion of the hypothalamic decapeptide, gonadotropin-releasing hormone (GnRH), which operates as a major hierarchical element of this system, driving the secretory pulses of pituitary gonadotropins, luteinizing hormone (LH) and follicle-stimulating hormone (FSH), that in turn control gonadal function (1, 2). Among the myriad of central regulatory signals converging on GnRH neurons, kisspeptins, encoded by the Kiss1 gene and operating via the G-protein-coupled receptor Gpr54, have been recognized in the past decades as major stimulators of GnRH neurosecretion and as essential elements in the central control of puberty onset, sex steroid feedbacks, fertility, and, possibly, sexual behaviors (2-5).

Tachykinins (TACs) have also been identified as key elements in the central control of $\mathrm{GnRH} / \mathrm{LH}$ secretion in different species (6-9). The mammalian TAC family is composed of a number of neuropeptides, which include substance $P$ (SP), neurokinin A (NKA), and neurokinin B (NKB), as well as 
the extended forms of NKA, neuropeptide K (NPK) and $\gamma$ $(\mathrm{NP} \gamma)$, all having the characteristic C-terminal sequence, Phe-X-Gly-Leu-Met- $\mathrm{NH}_{2}(10,11)$. TACs are widely distributed throughout the central nervous system and periphery, playing a diverse array of functions that include modulation of neural activity, control of behavioral responses, maintenance of fertility, vasodilation, and smooth muscle contraction (10-12). To date, most attention in TAC research has focused on the biological effects of SP, NKA (both encoded by a single gene, Tac1 in rodents), and NKB (encoded by Tac2 in rodents), which bind preferentially to the G-proteincoupled receptors NK1R (encoded by Tacr1), NK2R (encoded by Tacr 2 ), and NK3R (encoded by Tacr3), respectively (10, 11, 13). Notwithstanding this ligand preference, some degree of cross reactivity between TACs and their receptors has been documented $(14,15)$. Thus, while TAC effects on LH release were blunted by central administration of an antagonist for all three receptors, administration of each TAC receptor antagonist alone had no effect on LH secretion in rats (8). In the same vein, full blockade of NKB actions on GnRH neuron firing was only achieved when concomitant suppression of all three TAC receptors was applied (14).

A majority of the studies reported to date on the roles of TACs in the control of GnRH/LH secretion have focused on the role of the NKB/NK3R system, as initial reports documented that inactivating mutations of the genes encoding NKB (TAC3) or NK3R (TACR3) in humans were associated with hypogonadotropic hypogonadism $(\mathrm{HH})$ and lack of puberty $(16,17)$. Interestingly, studies in rodents, sheep, monkeys, and humans have documented the coexpression of NKB in a substantial fraction of Kiss1 neurons located in the hypothalamic arcuate nucleus (ARC) (18-21); this population was termed KNDy, as they coexpress not only Kiss1 and $N$ KB but, to a variable degree, also Dynorphin (Dyn) (22). This defines a sort of oscillatory network, in which NKB activates and Dyn preferentially inhibits kisspeptin output to GnRH neurons (2, 7, 23); a neuronal setup that has been recently proven as an essential component of the GnRH pulse generator $(24,25)$. In good agreement, the stimulatory effects of the NKB agonist, senktide, were absent in Gpr54 null mice, suggesting mediation via kisspeptin signaling $(3,7,26)$.

Despite the reported human phenotype, genetic inactivation of Tac2 or Tacr 3 caused a less severe reproductive impairment in mice $(27,28)$. For instance, Tacr3 null female mice were only subfertile, whereas Tacr3-deficient males attained fertility. Similarly, $\mathrm{Tac}^{-/-}$female mice displayed delayed puberty but were otherwise fertile at adulthood, while males showed no overt reproductive defects. Altogether, these features resemble the human phenotype of some mutations of the NKB pathway, in which partial or complete reversal of their HH state has been reported (29). As a plausible interpretation, it has been proposed that, due to some degree of redundancy among the TAC pathways, SP/NK1R and/or NKA/NK2R signaling may compensate for the congenital absence of NKB actions, thereby preserving fertility in mice and permitting reversal of the hypogonadal state in humans, with genetic inactivation of the NKB pathway. This redundancy has been recently documented by phenotypic analyses on double Tac1/Tac2 KO mice, which displayed more profound reproductive defects than mice lacking one of the two Tac genes (30).
Although less characterized than the NKB/NK3R system, compelling evidence supports the involvement of SP/NK1R in the control of reproduction (15). This TAC system seemingly has variable effects on $\mathrm{LH}$ secretion depending on the species, so that NK1R agonists stimulated LH secretion in intact male mice, as well as in ovariectomized (OVX) female mice, supplemented or not with estradiol, while in adult rats, no LH responses to NK1R activation were detected (7, 31). Recent evidence has further documented the role of the SP/NK1R pathway in the modulation of puberty and fertility. Thus, in prepubertal female mice, central injection of an NK1R agonist elicited gonadotropin secretion, while Tac1 KO female mice displayed delayed puberty and subfertility (32). Notably, SP/NK1R signaling seems to be also involved in the control of sexual behavior, as injection of SP into the midbrain central gray facilitated lordosis (33), and Tacr1 KO male mice showed decreased pheromone-induced sexual approach behavior (34).

In contrast, the physiological roles of the NKA/NK2R system in the control of the reproductive axis remain largely unexplored. Although NK2R does not seem to be expressed in Kiss1 (or GnRH) neurons, NKA agonists have been found to be capable of regulating gonadotropin secretion in a sex steroid- and kisspeptin-dependent manner, with stimulatory and inhibitory actions on LH release being reported (7, 35, 36). More recently, a putative role for the NKA/NK2R pathway in the control of puberty and the gonadotropic axis was suggested by pharmacological studies showing that activation of NK2R in prepubertal female mice advances puberty onset, while it stimulates LH release in adulthood (36). In the same line, the delayed puberty and subfertility seen in Tac1 null mice might be caused, at least partially, by the lack of endogenous NKA, since this neuropeptide, together with SP, is ablated in this mouse line (32). Nonetheless, the overlapping functions of other TAC pathways, the partial promiscuity at the receptor level, and the lack of more incisive genetic models have prevented further elucidation of the specific biological roles and actual physiological relevance of NK2R signaling in the control of the reproductive axis. In the present study, we aimed to characterize more deeply the role of NK2R in the regulation of the reproductive axis by analyzing a novel $\mathrm{TaCr}^{-1-}$ mouse line.

\section{MATERIALS AND METHODS}

\section{Ethics Statement}

The experiments and animal protocols included in this study were approved by the Ethical Committee of the University of Córdoba; all experiments were conducted in accordance with the European Union (EU) normative for the use and care of experimental animals (EU Directive 2010/63/UE, September 2010).

\section{Animals}

Mice were housed in the Animal Service for Experimentation (SAEX) of the University of Córdoba. All animals were maintained under a 12-h light/dark cycle, at standard temperature $\left(22 \pm 2^{\circ} \mathrm{C}\right)$, and given ad libitum access to standard laboratory mice chow (A04, Panlab) and water, unless mentioned otherwise. The day the litters were born was considered as 
day 1 of age (PND1); animals were weaned at PND21. For the diet-induced obesity studies, adult mice were fed with a highfat diet (HFD, Ref. No, D12451; Research Diets, New Brunswick, NJ) with $45 \%, 20 \%$, and $35 \%$ calories from fat, protein, and carbohydrate, respectively, for a period of $10 \mathrm{wk}$.

\section{Tacr2 $^{-1-}$ Mouse Line}

Tacr2 knockout mice (named hereafter $\mathrm{Tacr}^{-/-}$) were obtained from the UCDavis KOMP Repository [referred to there as $t m 1(K O M P) W t s i$, tm1(KOMP)Mbp]. The strategy for disrupting the Tacr2 gene was to replace exon 2 with a LacZ/ neo cassette. Genotyping was conducted by final PCR analyses on genomic DNA isolated from mouse ear tissue. Two PCR amplifications per mouse were set; one specific to detect the wild-type (WT) allele (210 base pair, bp) and another one for the disruption of Tacr 2 allele ( $875 \mathrm{bp}$ ). The primers used for detection of the WT allele were as follows: 5'-CTC CTG ACC ATC ACC CAA TCC-3' and 5'-GGC TGG AAA GGG TGG ACA ATG G-3', while the primers used to detect the mutated allele were as follows: 5'-TGG ATT CAT CGA CTG TGG CCG3 ' and 5'-TGA GTC TCA AGC CTA GCT CCC TAG-3'.

\section{Drugs}

The NK1R agonist (GR 73632), NK2R agonist (GR 64349), NK3R agonist (senktide), and NK3R antagonist (SB 222200) were purchased from Tocris Bioscience (Bristol, UK). Of note, GR 64349 has been reported as a potent and selective NK2R agonist, displaying $>1,000$-fold and $>300$-fold selectivity over NK1R and NK3R, respectively (37), and $>10$-fold higher selectivity for NK2R than the endogenous ligand, NKA (38); hence, GR 64349 can be considered as a bona fide NK2R agonist. Kisspeptin (110-119)-NH2 (referred as kisspeptin-10 or Kp-10) was obtained from Phoenix Pharmaceuticals Inc. (Burlingame, CA). 17 $\beta$-estradiol (E2), glucose, and insulin were obtained from Sigma-Aldrich (St. Louis, MO). Kp-10, NK2R agonist, NK1R agonist, and senktide were dissolved in physiological saline ( $0.9 \%$ sodium chloride); E2 was dissolved in olive oil; glucose and insulin were dissolved in water for injections; and the NK3R antagonist was dissolved in 50\% dimethyl sulfoxide (DMSO). The doses for the different drugs, administered via the intracerebroventricular route, were as follows: Kp-10 (50 pmol), GR 73632 (3 nmol), GR 64349 (600 pmol), senktide $(600 \mathrm{pmol})$, and SB222200 $(7 \mathrm{nmol})$. The drugs were administered in a final volume of $5 \mu \mathrm{L}$, to allow accurate dose infusion of the compounds without adverse effects, in line with the extensive experience of our group $(3,39-41)$. The intraperitoneal bolus of glucose used in the glucose tolerance test (GTT) was $2 \mathrm{~g} / \mathrm{kg}$ of body weight (BW) per mouse, and the bolus of insulin injected intraperitoneal for the insulin tolerance test (ITT) was 0.75 international units (IU).

\section{Experimental Design and Analytical Methods}

As general rule, assignment of animals to the different experimental groups was done by stratified randomization within each genotype. However, due to operational reasons, groups were not blinded to the experimenters.

\section{General procedures and LH assay.}

For pharmacological studies, intracerebroventricular injections were conducted. Cannulas (INTRADEMIC polyethylene tubing, Becton Dickinson, Sparks, MD) were implanted, 2-3 days before the initiaition of the experiments, into the rodent brain at $2 \mathrm{~mm}$ depth, with coordenates of $1 \mathrm{~mm}$ posterior and $1.2 \mathrm{~mm}$ lateral to bregma, to allow delivery into the lateral ventricle; a procedure that is well validated, based on postmortem necropsy, and routinely used in our research team $(3,39-41)$. For cannula implantation, mice were anesthetized with isoflurane.

For hormone (LH) assays, blood samples were obtained from the mouse tail at at the designated times after intracerebroventricular injection of the agonists. For each sample, $4 \mu \mathrm{L}$ of whole blood was diluted in $46 \mu \mathrm{L}$ of $0.1 \mathrm{M}$ phosphatebuffered saline (PBS) with $0.05 \%$ Tween 20 , snap-frozen on dry ice, and stored at $-80^{\circ} \mathrm{C}$ until the assay using a supersensitive LH ELISA (42). Mice were handled (5-10 min per animal) every day for $3 \mathrm{wk}$ before blood sampling, to habituate them for tail-tip bleeding. For blood sample collection, mice were restrained in a cardboard tube every 5-min interval during $3 \mathrm{~h}$ for taking serial tail-tip blood samples. Samples were stored at $-80^{\circ} \mathrm{C}$ for the subsequent hormone assay. For studies involving real-time PCR (RT-PCR), hypothalami were dissected out, immediately upon the decapitation of the animals, frozen in liquid nitrogen, and stored at $-80^{\circ} \mathrm{C}$ until used for molecular analyses.

\section{Phenotypic evaluation of pubertal maturation and fertility of Tacr2 null mice.}

After weaning, 3-wk-old mice were checked for phenotypic markers of puberty onset on a daily basis. Somatic and reproductive indices of pubertal development were monitored, including body weight (BW), age of vaginal opening (VO), and balano-preputial separation (BPS), the two latter being considered external markers of puberty onset in female and male rodents, respectively. Once vaginal opening occurred, vaginal cytology was performed daily to identify the age of the first estrus (FE), a marker of first ovulation in female rodents.

\section{Reproductive assessment of adult Tacr2 null mice.}

Adult virgin female mice were monitored daily by vaginal cytology for at least 3-4 wk to characterize estrous cyclicity. Fertility studies were performed by breeding adult Tacr $2 \mathrm{KO}$ mice of either sex with fertile WT counterparts. Litter size, time lapse until successful pregnancy in females, and pregnancy rates were monitored.

\section{Pharmacological studies in adult control and Tacr2 null mice.}

To assess the role of NK2R signaling in the control of the gonadotropic axis, we analyzed time-course LH responses to intracerebroventricular administration of the NK2R agonist GR 64349 in control and $\mathrm{Tacr}^{-1-}$ male and female mice, and compared those with LH secretory responses induced by the NK1R and NK3R agonists, as well as Kp-10. The total number of animals used in the pharmacological experiments, per genotype and sex, was as follows: females, control: $n=17$; Tacr2 $2^{-/-}: n=17$; males: control: $n=22$; Tacr2 ${ }^{-1-}$ : $n=27$. Subsets of mice from the available pool of mice were injected with the NK2R agonist ( $600 \mathrm{pmol})$, the NK3R agonist $(600 \mathrm{pmol})$, the NK1R agonist $(3 \mathrm{nmol})$, or $\mathrm{Kp}-10(50 \mathrm{pmol})$. To optimize animal use, minimizing the total number of 
mice needed while securing sufficient group sizes for robust statistical analyses, repeated testing was allowed, under the following premises: 1 ) time-course analyses were run in parallel for both genotypes; 2) animals were allowed to recover for at least $72 \mathrm{~h}$ between tests; and 3) animals were not included in more than three tests and were evenly distributed across the tests to allow grossly similar recovery times. In each test, blood samples were collected before injections (to set basal reference levels) and at 15, 30, and 60 after intracerebroventricular administration. Doses and routes of administration were selected on the basis of previous references $(7,36)$. Details of the number of individuals included in each pharmacological test are included in Supplemental Table S1; all Supplemental material is available at http://dx. doi.org/10.17632/fktzgbbdpr.1.

In an additional experiment, we intended to assess $\mathrm{LH}$ responses to the NK2R agonist GR 64349 after pharmacological NK3R blockade, using the selective antagonist SB222200 $(7 \mathrm{nmol} /$ mouse in $5 \mu \mathrm{L}$ icv). To this end, adult WT and $\mathrm{Tacr}^{-1-}$ male mice were pretreated with SB222200 $60 \mathrm{~min}$ before the intracerebroventricular administration of GR 64349 (600 pmol). This test was conducted selectively in males, since our initial analyses revealed that LH responses to GR 64349 were already blunted in $\mathrm{Tacr}^{-1-}$ females but not significantly altered in $\mathrm{Tacr}^{-/-}$male mice. Blood samples were collected before (basal) and at 15 and 60 min after SB222200 administration and at 15, 30, and 60 min after the injection of GR 64349. For further details, see Supplemental Table S1.

\section{Gene expression analyses in the mediobasal hypothalamus of Tacr 2 null mice.}

To confirm effective ablation of NK2R and to determine whether the $\mathrm{Tacr} 2^{-/-}$mice display compensatory changes in expression of other key elements in the central control of the reproductive axis, we measured the expression levels of the mRNAs encoding NK1R (Tacr1), NK2R (Tacr2), NK3R (Tacr3), NKB (Tac2), kisspeptin (Kiss1), GnRH (Gnrh1), and dynorphin (Pdyn) in the mediobasal hypothalamus (MBH) of WT $(n=5-7)$ and Tacr2 KO $(n=5-7)$ female and male mice. To this end, total RNA from the MBH was isolated using the Favorgen Tissue Total RNA Extraction Mini Kit (FATRK001) according to the manufacturer's protocol. RNA was quantified using the Nanodrop ND-1000 v3.5.2 spectrophotometer (Nanodrop Technology, Cambridge, UK), and $0.5 \mu \mathrm{g}$ of RNA was reverse transcribed using the iScriptcDNA synthesis kit (Bio-Rad Laboratories Inc.). Quantitative real-time PCR assays were performed on a CFX96 Touch Real-Time PCR Detection System (Bio-Rad Laboratories Inc.) and using SYBR Green qPCR Master Mix (Promega Corporation). The primer pairs, cycling conditions, and amplicon product size used are indicated in Supplemental Table S2.

\section{Assessment of LH pulsatile secretion in Tacr2 null female mice.}

Assessment of pulsatile LH secretion was conducted in control and Tacr $2^{-/-}$female mice $(n=8-10)$. To avoid the potential confounding factor posed by dynamic fluctuations of endogenous estrogen levels, 4-5-month-old female mice were subjected to bilateral ovariectomy (OVX) under isoflurane anesthesia, followed by 2 -wk replacement with physiological levels of estradiol $\left(\mathrm{E}_{2}\right)$, according to previous references $(20,43)$. After the standard habituation period (see Genaral procedures and LH assay), serial tail-tip blood samples were collected at 5-min intervals during $3 \mathrm{~h}$. The number of pulses and the basal and mean LH levels were measured. For OVX $+\mathrm{E}_{2}$ mice, $\mathrm{LH}$ pulses were defined as those displaying an increase of $20 \%$ over the preceding two points; formula was adapted from previous references $(24,44)$. Basal LH levels were calculated using the mean of the values preceding each peak, whereas mean LH levels were estimated as the arithmetic mean of all $\mathrm{LH}$ values over the study period.

\section{Assessment of $L H$ responses to ovariectomy in Tacr2 null female mice.}

To investigate the role of NK2R signaling in mediating the negative feedback effects of ovarian steroids on LH release, control and $\mathrm{TaCr}^{-/-}$female mice were subjected to bilateral ovariectomy (OVX) via abdominal incision under light isoflurane anesthesia. Blood samples were collected before surgery and 2 and 7 days after bilateral OVX. Body weight changes were monitored in parallel.

\section{Analysis of the effect of fasting on $\mathrm{LH}$ secretion in Tacr2 null mice.}

To determine whether Tacr2 null mice display alterations in LH responses to nutritional stress caused by food deprivation, control and Tacr2 ${ }^{-/-}$male mice were subjected to fasting for a total period of $24 \mathrm{~h}$. Blood samples were collected before and at 12, 18, and $24 \mathrm{~h}$ of fasting. Body weight changes were monitored in parallel.

\section{Evaluation of metabolic parameters in Tacr2 null mice.}

To assess the role of NK2R signaling in the control of key metabolic parameters, we carried out body weight and composition analyses, in both males and females, as well as glucose tolerance tests (GTT) and insulin tolerance tests (ITT) in adult WT and Tacr2 $2^{-/}$male mice, fed with either standard (low-fat content; $<8.5 \%$ ) chow or a $45 \%$ HFD. In addition, noninvasive measures of food intake, energy expenditure (EE), respiratory quotient (RQ), and locomotor activity were conducted in adult WT and Tacr2 null mice of both sexes fed with standard chow. For analyzing the body composition, quantitative magnetic resonance (QMR) imaging using the EchoMRI 700 analyzer (Houston, TX, software v.2.0) was performed. For GTT and ITT, male mice $(n=5-13)$ were fasted for $4 \mathrm{~h}$ and subsequently received an intraperitoneal bolus of glucose and/or insulin, as described in previous sections. Glucose concentrations from tail-tip bleeding were monitored before (basal) and at 20,60, and $120 \mathrm{~min}$ after ip injections, using a handheld glucometer (ACCU-CHECK Aviva; Roche Diagnostics). For GTT and ITT, the total area under the curve (AUC) of glucose concentrations was determined by the trapezoidal method, during the 120 min following glucose and/or insulin administration.

To determine the EE and RQ, after a 24-h acclimation to the metabolism cages, mice were monitored for a further $24 \mathrm{~h}$ with ad libitum access to standard laboratory chow and water. Animal $\mathrm{O}_{2}$ consumption rate $\left(\mathrm{VO}_{2}\right)$ and $\mathrm{CO}_{2}$ production rate $\left(\mathrm{VCO}_{2}\right)$ were measured every 40 min, allowing calculation of EE and RQ. Locomotor activity of the animal and measurement of food intake were recorded every $3 \mathrm{~min}$. 
Finally, systolic blood pressure was measured in control and Tacr $^{-/-}$male and female mice by using a tail-cuff sphygmomanometer.

\section{Female urine sniffing test.}

To examine whether Tacr2 KO male mice can distinguish between a sex odor (female urine, $10 \mu \mathrm{L}$ ) and nonsocial odor (water, $10 \mu \mathrm{L}$ ) on cotton pads located in opposite corners of the cage in comparison with WT male $(n=17-20)$, the female urine sniffing test (FUST) was performed, as adapted from previous references $(45,46)$. In short, urine samples were collected from mature WT female mice from different cages and pooled for decreasing variability in sniffing time. Mice were individually placed into the Social Box (Panlab -LE894-) and habituated for $5 \mathrm{~min}$. After the acclimation period, mice were presented to the social and nonsocial odors. The time spent sniffing each cotton pad was recorded using Debut Video Capture Software (NCH Software) and digitally determined by using a video tracking software (EthoVision XT, Noldus). The open-field cage was cleaned with $70 \%$ ethanol followed by double-distilled water after each test.

\section{Three-chamber sex preference test.}

To test sex preference, WT and Tacr2 null female and male mice $(n=9-15)$ were individually put into the Social Box and allowed to acclimate for $5 \mathrm{~min}$. After the habituation stage, mice were allowed to explore, during $10 \mathrm{~min}$, the arena where nonfamiliar female and male mice were placed in opposite corners of the cage into a closed wire basket. The nonfamiliar mice used in the sex preference test were previously habituated to the wire basket for 3 days. The time spent by the mouse in each chamber and in close interaction was recorded using Debut Video Capture Software (NCH Software) and calculated automatically by a video tracking software (EthoVision XT, Noldus). The open-field cage was cleaned with $70 \%$ ethanol followed by double-distilled water after each test.

\section{Presentation of Data and Statistical Analyses}

Statistical analyses were performed using Prism software (GraphPad Prism). All data are presented as means \pm SE. An unpaired $t$ test was applied for the assessment of differences between two groups and ANOVA followed by post hoc Bonferroni tests for comparisons of more than two groups. The significance level was set at $P \leq 0.05$, and different letters or asterisks indicate statistical significance. Sample size was adjusted based on animal availability (for each genotype and sex) and previous experience of our group in analogous studies addressing molecular and neuroendocrine aspects of the central mechanisms controlling reproduction and metabolism. These calculations were assisted by power analyses performed using values of standard deviation that we get usually when assessing parameters similar to those of this study. All relevant sample sizes are provided in the MATERIALS AND METHODS, figure legends, and in Supplemental Table S1.

\section{Data Availability Statement}

The authors declare that the data supporting the findings of this study are included in this article and its supplementary information files. All relevant original data are available from the corresponding authors, upon request.

\section{RESULTS}

\section{LH Responses to NK2R Activation in Control and Adult Tacr2 $^{-1-}$ Mice: Comparison with Other TACs and Kp}

We explored time-course LH responses to the agonist of NK2R GR 64349 in $\mathrm{Tacr}^{-/-}$mice of both sexes, and their corresponding controls, and compared these responses with those induced by agonists of NK1R (GR 73632) and NK3R (senktide), as well as kisspeptin-10 (Kp-10). In line with genotyping results, male and female $\mathrm{TaCr}^{-/-}$mice displayed absent Tacr 2 mRNA expression in the hypothalamus (Supplemental Fig. S1), which was present in control mice, validating the selective lack of NK2R in our model. In contrast, hypothalamic expression of a number of genes encoding key regulators of the reproductive axis, including Tacr1, Tacr3, Kiss1, Gnrh1, Tac2, and Pdyn, was detectable in the hypothalamus of $\mathrm{Tacr}^{-/-}$mice of both sexes, at levels comparable with those seen in control mice (Supplemental Fig. S1).

All the analogs tested evoked significant elevations of serum LH levels in control male and female mice, in line with previous literature $(3,7,36)$, with peak values being achieved between 15 and $30 \mathrm{~min}$ after injection. In fact, comparison of the magnitude of such responses at $30 \mathrm{~min}$ after intracerebroventricular administration showed that activation of NK2R with GR 64349 evoked a significant twofold elevation of serum LH levels over basal values, which was grossly similar in magnitude to the responses elicited by NK1R and NK3R agonists, in both male and female mice. In turn, adult mice of both sexes responded to a bolus of a low dose of Kp10 with a robust increase in LH levels, 30 min after intracerebroventricular injection, which was significantly higher than any of the LH responses to the individual NKR agonists (Supplemental Fig. S2).

In $\mathrm{Tacr}^{-/-}$female mice, LH responses to the NK2R agonist GR 64349 were detectable but significantly attenuated versus controls, as defined by lower LH levels at 15 and 30 min after agonist injection (Fig. $1 A$ ), and the overall decrease of integral ( $\triangle \mathrm{AUC}$ ) LH responses for the 60 -min period following administration of GR 64349 (Fig. 1C). In contrast, LH responses to the other TAC receptor agonists and $\mathrm{Kp}-10$ were not affected by Tacr 2 ablation and were similar in magnitude to those of control female mice (Fig. 1A). Tacr2 null male mice displayed a grossly conserved LH responsiveness to the NK2R agonist, as denoted in time-course and integral analyses (Fig. 1, $B$ and $C$ ), except for a very modest decrease in serum LH levels over corresponding control values at 60 min after injection of GR 64349. In addition, the stimulatory effects of the other TAC receptor agonists and $\mathrm{Kp}-10$ on $\mathrm{LH}$ secretion were similar in $\mathrm{Tacr}^{-/-}$and control male mice (Fig. 1B).

The intriguing conservation of LH responses to the NK2R agonist in male mice congenitally lacking this receptor prompted us to explore the potential interplay between NK2R and other TAC pathways in this model. Of note, this phenomenon was studied selectively in males, as $\mathrm{Tacr}^{-/-}$ females were already displaying reduced $\mathrm{LH}$ responses to 
A
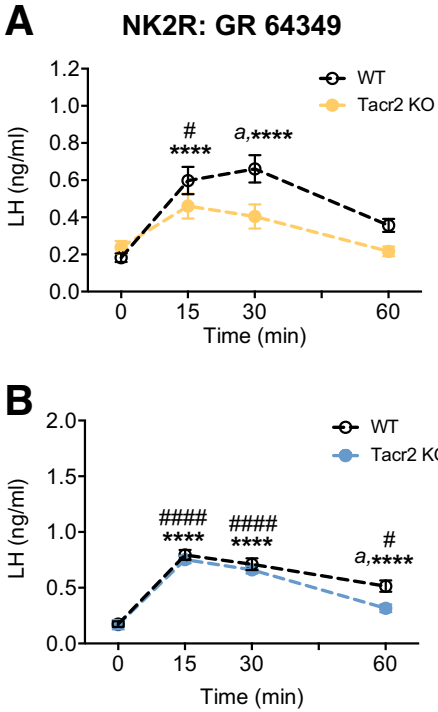

C

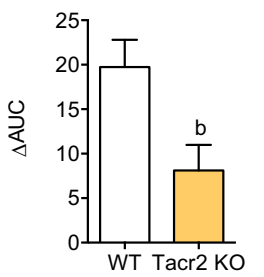

NK1R: GR 73632
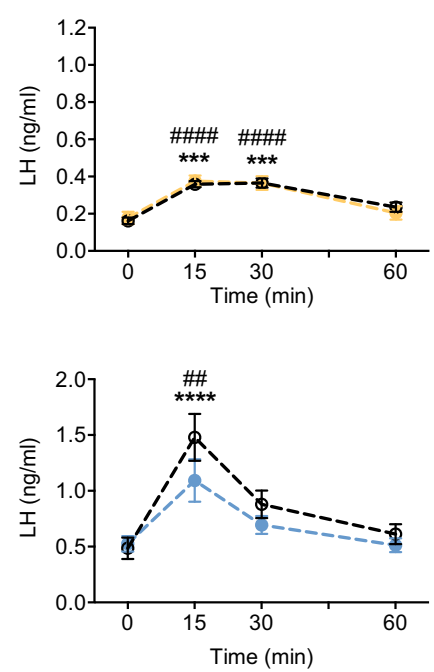

D
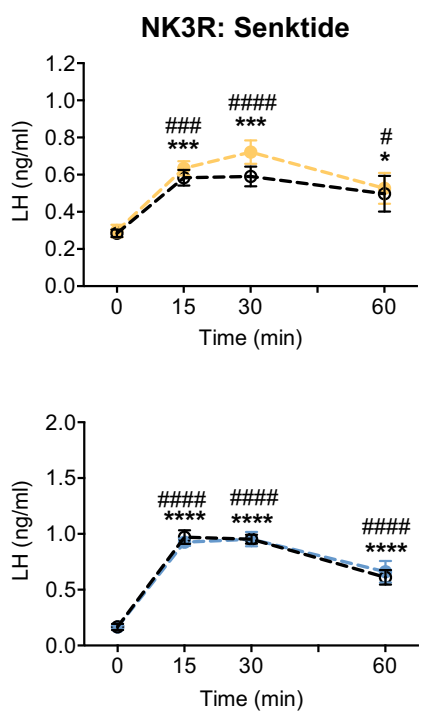

Gpr54: Kp-10
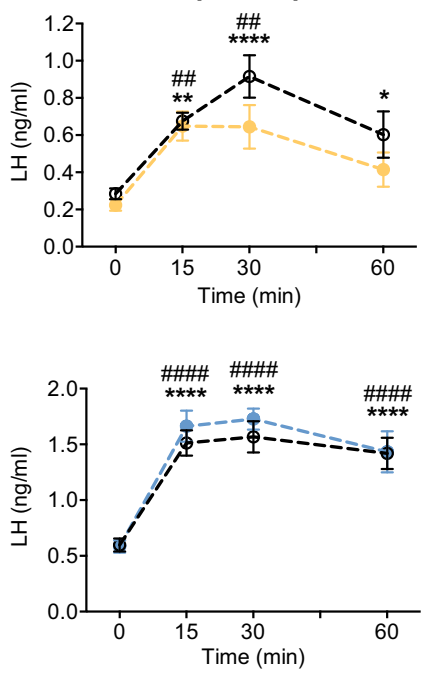

\section{$\triangle A U C$ (to NK3R-ANT}
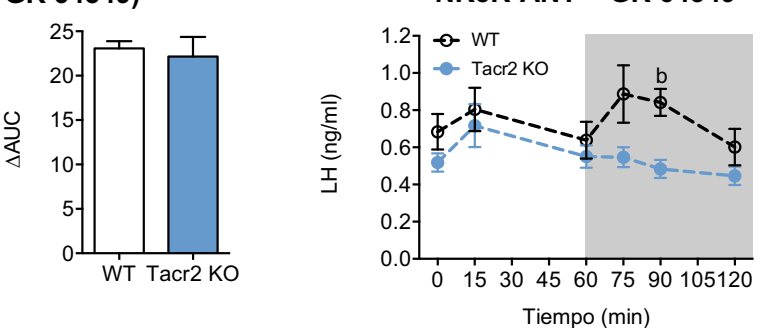

+ GR 64349)

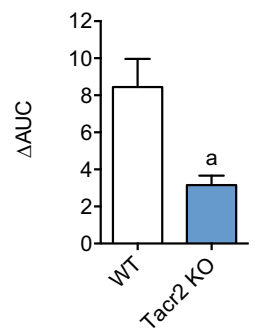

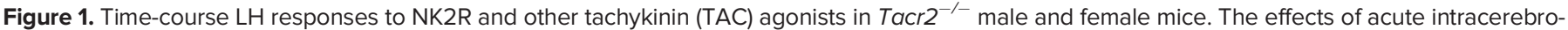

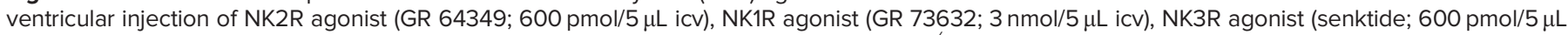

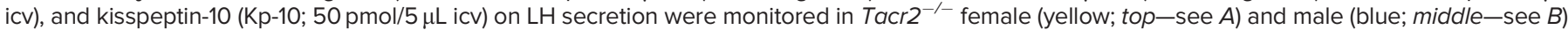

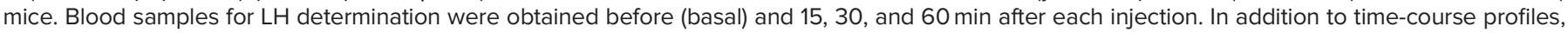

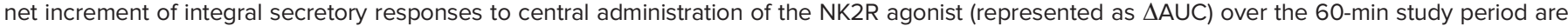

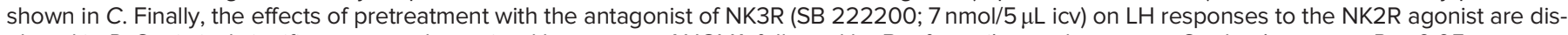

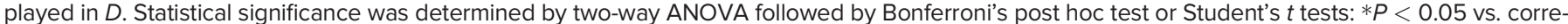

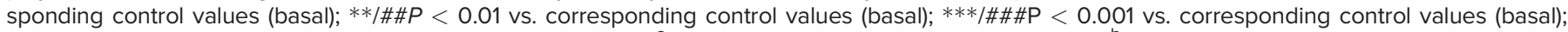

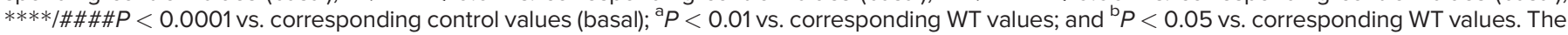
number of animals used in each hormonal test is indicated in Supplemental Table S1. AUC, area under the curve.

the NK2R agonist, suggesting a lower degree of redundancy. In detail, male Tacr $^{-/-}$and control mice were pretreated with the NK3R antagonist SB 222200, and after $60 \mathrm{~min}$, the animals were intracerebroventricularly injected with an effective dose of the NK2R agonist GR 64349. In line with our previous data, control mice pretreated with the NK3R antagonist displayed significant LH responses to NK2R activation. In contrast, $\mathrm{LH}$ responses to the NK2R agonist GR 64349 were fully prevented by the concomitant blockade of NK3R in Tacr2 ${ }^{-/-}$mice (Fig. 1D).

\section{Tacr $^{-/-}$Mice Display Preserved Puberty and Fertility but Altered LH Pulsatility}

Puberty onset and reproductive parameters were monitored in Tacr2 null mice of both sexes. Analysis of phenotypic markers of puberty revealed normal pubertal timing in Tacr $^{-/-}$male and female mice, as denoted by the conserved ages of vaginal opening (VO) and first estrus (FE) in females (Fig. 2, B-E) and balano-preputial separation (BPS) in males (Fig. 2, $G$ and $H$ ). In addition, body weight (BW) gain during the pubertal transition was similar in $\mathrm{KO}$ and control mice of both sexes (Fig. 2, $A$ and $F$ ).

Additional parameters of the functioning of the reproductive axis were monitored in Tacr $2^{-/-}$female mice. In detail, pulsatile LH secretion was assessed over a 180-min period, using frequent 5-min blood sampling and a super-sensitive LH ELISA. In addition, estrous cyclicity and fecundity were studied; the latter also in Tacr2 null males. To avoid the potential interference of fluctuations of endogenous estrogen levels, LH pulsatility was evaluated in female mice of both genotypes following bilateral ovariectomy (OVX) and replacement with a physiological dose of estradiol $\left(\mathrm{E}_{2}\right)$. In this model of clamped $\mathrm{E}_{2}$ concentrations, basal and mean $\mathrm{LH}$ levels were significantly lower in Tacr $2^{-/-}$female mice. In contrast, the number of pulses was not significantly altered in null mice; if any, a trend for a higher number of pulses over the 180-min period was observed (Fig. 3, $A$ and $B$ ). Despite these alterations in LH pulsatility, estrous cyclicity was fully preserved in $\mathrm{Tacr}^{-/}$female mice, without any differences being observed in terms of total cycle length or stage distribution/ 

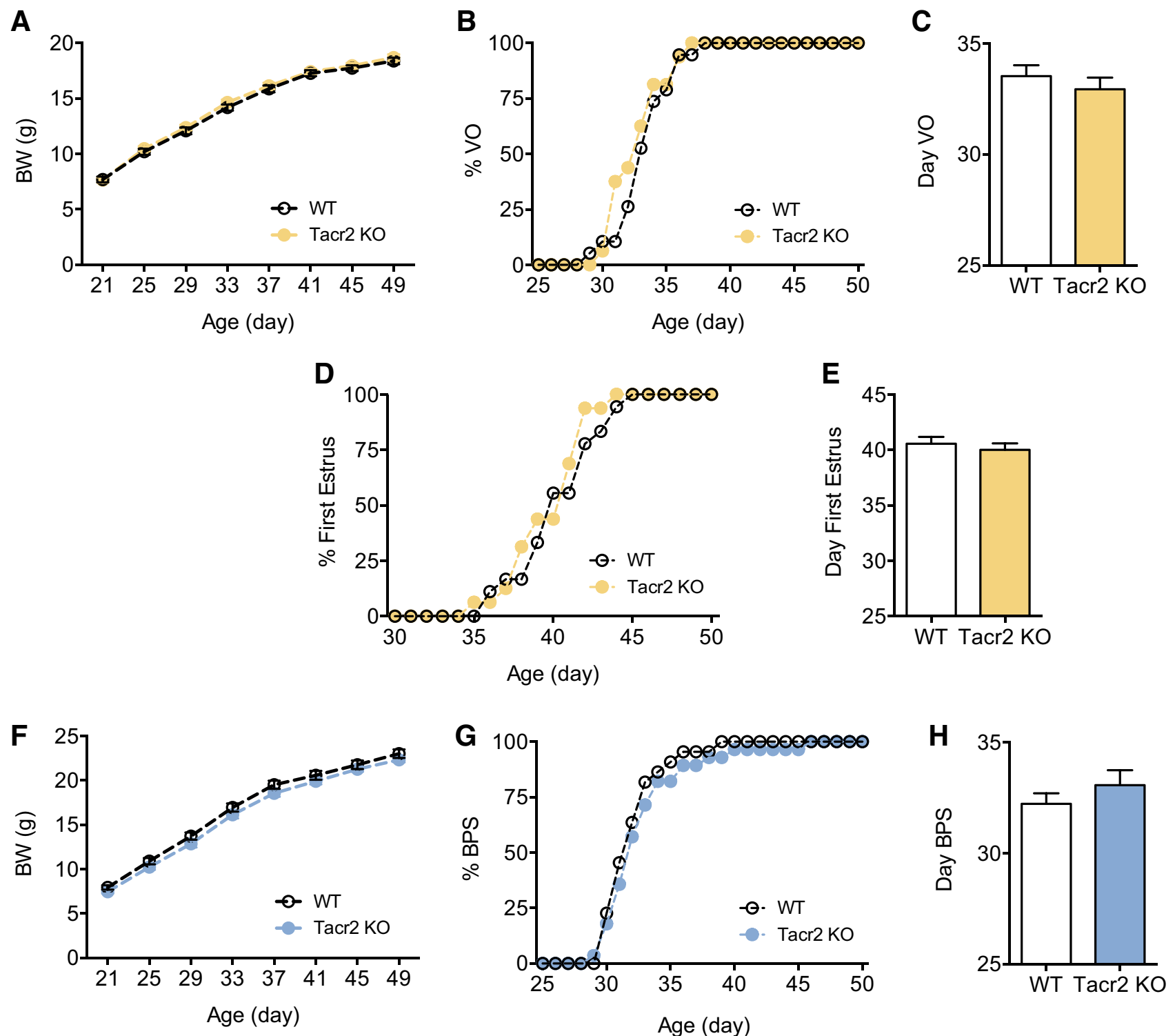

Figure 2. Indices of pubertal maturation in Tacr $2^{-1-}$ male and female mice. A: evolution of body weight (BW) gain from weaning (PND21) to adulthood is presented from wild-type (WT) (white) and $\operatorname{Tacr}^{-\prime-}$ (yellow) female mice (WT: $\left.n=18 ; \operatorname{Tacr}^{-\prime-}: n=16\right)$. B: the accumulated percentage of female mice displaying vaginal opening (VO) during the period of 3 wk after weaning is shown; the average age of $\mathrm{VO}$ is presented in $C$. The accumulated percentage of female mice displaying first estrus (FE) and the average age of FE are shown in $D$ and $E$, respectively. In addition, evolution of BW gain from weaning (PND21) to adulthood is presented from WT (white) and Tacr2 ${ }^{-1-}$ (blue) male mice (WT: $n=22 ;$ Tacr2 KO: $n=28$ ) in presented in $F$. G: the accumulated percentage of male mice displaying balano-preputial separation (BPS) during the period of $3 \mathrm{wk}$ after weaning is shown, while the average age of BPS is presented in $\mathrm{H}$.

frequency (Fig. 3, $C-E$ ). Finally, fertility tests were conducted by crossing control or $\mathrm{TaCr}^{-/-}$mice of either sex with control mice of the opposite sex. A nonsignificant trend for increased breeding intervals was detected, especially for null females, although due to inherent variability, this did not reach statistical significance (Fig. 3, $F$ and $G$ ).

In addition, we monitored changes in LH levels in $\mathrm{Tacr}^{-/-}$mice in response to hormonal or nutritional manipulations, previously reported to modify LH secretion (47), such as ovariectomy and short-term fasting. In control mice, LH concentrations rose shortly after OVX, with a significant elevation already at day 2 after surgery, and massively increased LH concentrations being detected by 1 wk after OVX. A similar profile was detected in $\mathrm{Tacr}^{-/-}$ mice; yet, the elevation in LH levels observed at day 2 postOVX was not statistically significant over the corresponding basal levels (Fig. 4A). On the other hand, in male mice subjected to a 24-h period of fasting, LH levels were significantly lower in $\mathrm{TaCr}^{-1-}$ versus control mice, as evidenced by timecourse analyses and integral [area under the curve (AUC)] values (Fig. $4, B$ and $C$ ).

\section{Tacr2 $^{-1-}$ Mice Exhibit Normal Social Interaction and Sex Preference Behavior}

To address whether NK2R signaling participates in the control of key aspects of sexual behavior, as reported 

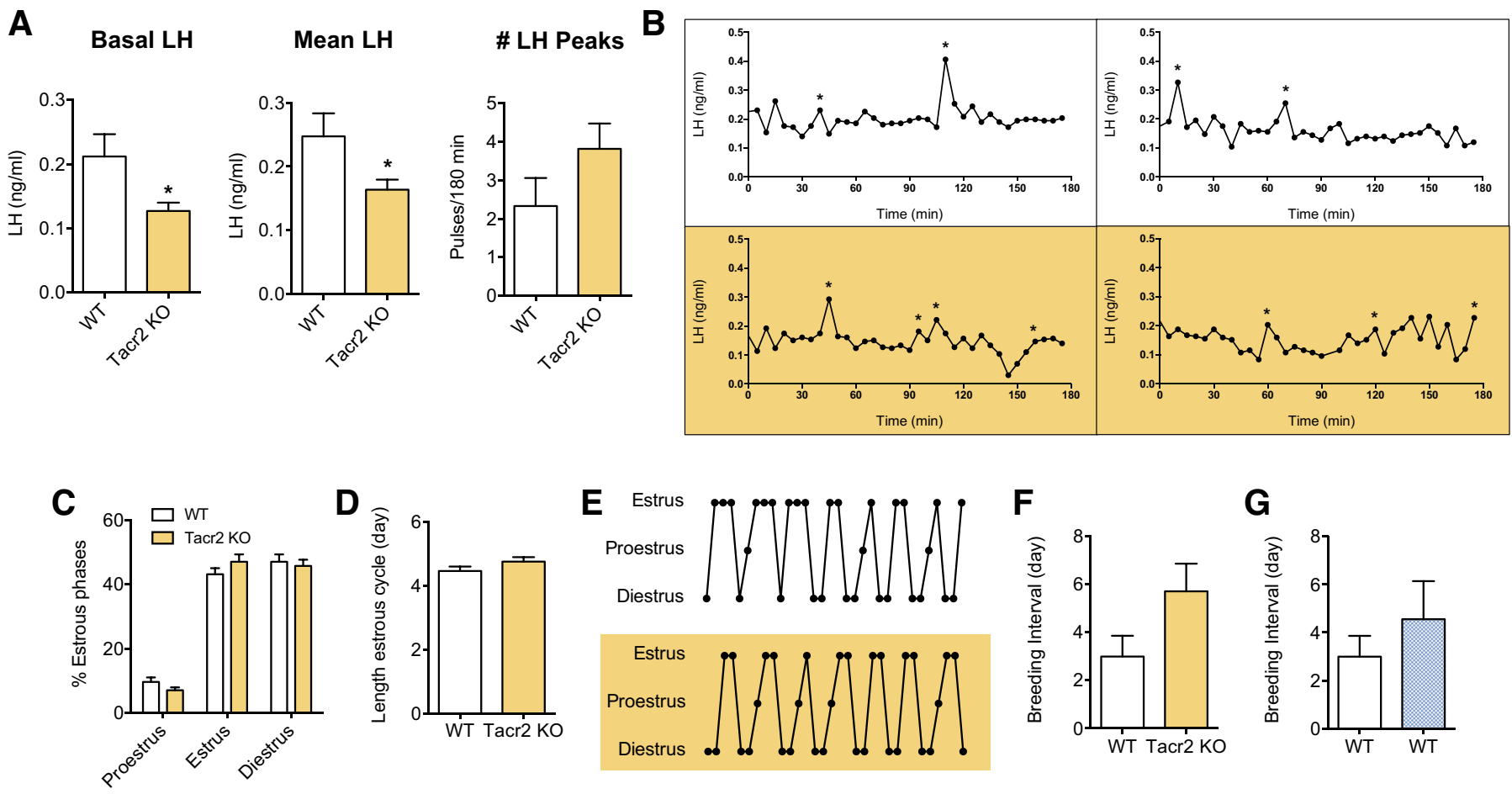

Figure 3. LH pulsatility and reproductive parameters in $\mathrm{TaCr}^{-1-}$ female mice. $A$ : histograms showing basal and mean $\mathrm{LH}$ levels, together with the numbers of LH pulses detected over a 3-h sampling period in wild-type (WT) and Tacr ${ }^{-/-}$mice, ovariectomized (OVX) and supplemented with estradiol ( $\left.E_{2}\right)$, are presented (WT: $n=8-9$; Tacr2 $\left.{ }^{-1-}: n=9-10\right)$. B: individual representative LH secretion profiles of OVX $+\mathrm{E}_{2}$ WT and Tacr2 ${ }^{-1-}$ mice are shown. In addition, histograms showing the percentual distribution of estrous cycle phases in WT and Tacr2 ${ }^{-1-}$ female mice are presented in $C$ (WT: $n=12$; Tacr2 KO: $n=12$ ). $D$ : the duration of the estrous cycle is displayed in WT and Tacr $2^{-1-}$ female mice. E: in addition, representative estrous cycle profiles in WT and Tacr2 $^{-1-}$ female mice are shown. F: finally, breeding intervals in WT (white; $n=8$ ) or Tacr2 ${ }^{-1-}$ (yellow; $n=14$ ) female mice mated with WT males are presented. G: for comparative purposes, the breeding intervals of WT female mice mated with WT (white; $n=8$ ) or Tacr $2 \mathrm{KO}$ (blue; $n=9$ ) male mice are also displayed. Statistical significance was determined by Student's $t$ tests: $* P<0.05$ vs WT values. In $B$, asterisks denote LH pulses.

previously for Tacr1 (34), we applied female urine sniffing and sex preference tests to Tacr2 ${ }^{-/-}$mice, following standard protocols. A three-chamber social sex preference test was applied to adult male and female null mice and their controls. In males, the stereotyped preference for the opposite (female) sex was documented; yet, no differences in any of the behavioral parameters explored, including velocity, distance moved, and time spent in the vicinity of the same or opposite-sex mouse, were noted between control and KO mice. Likewise, no differences in terms of sex preference and social behavior were found between control and $\mathrm{Tacr}^{-/-}$female mice (Fig. 5). In the same vein, sniffing tests revealed no defects in $\mathrm{Tacr}^{-/-}$ male mice in terms of preference for female urine odors; if any, better focusing for close interaction with the female versus neutral odor was observed in $\mathrm{TaCr}^{-/-}$null mice (Supplemental Fig. S3).

\section{Metabolic Profiles of Tacr2 ${ }^{-1-}$ Mice in Lean and Obesogenic Conditions}

To assess the impact of the lack of NK2R signaling on key metabolic parameters, we first monitored body weight gain in both female and male Tacr $2^{-/-}$mice, at 2 and 6 mo of age, which was similar to that of control littermates. In addition, body composition analyses at 2 mo of age confirmed similar fat and lean mass in Tacr2 null and control mice. Alike, energy expenditure (EE) and respiratory quotient (RQ), as well as total and nocturnal locomotor activity, were similar in control and Tacr2-deficient mice. No differences in terms of 24-h food intake and day/night feeding patterns were observed in $\mathrm{Tacr} 2^{-/-}$mice either. Finally, measurement of blood pressure by a tail-cuff sphygmomanometer demonstrated that Tacr2 null mice display values of systolic blood pressure similar to their control littermates. For further details, see Supplemental Figs. S4 and S5.

Additional metabolic analyses, addressing glucose homeostasis, were conducted in control and Tacr2 null mice, after challenge with an obesogenic diet. Note that due to animal availability, these analyses were conducted only in males. Exposure to an HFD for $10 \mathrm{wk}$ evoked a nonsignificant reduction of basal LH levels, which was associated with a significant increase in total body weight and basal glucose levels. Notably, whereas the rise of body weight induced by the HFD was similar between control and Tacr2 KO mice, the elevation of basal glucose levels was significantly higher in Tacr2 $^{-1-}$ males (Fig. 6, A-C). Likewise, GTT denoted that glucose intolerance was induced by HFD, but this parameter, as assessed by integral AUC glucose values over the 120-min period after the glucose bolus, reached statistical significance only in Tacr $2^{-/}$mice (Fig. 6, $D$ and $E$ ). In contrast, no major alterations in insulin sensitivity, measured by ITT, were detected in Tacr2 null mice, irrespective of the diet regimen (Fig. 6, $F$ and $G$ ). 

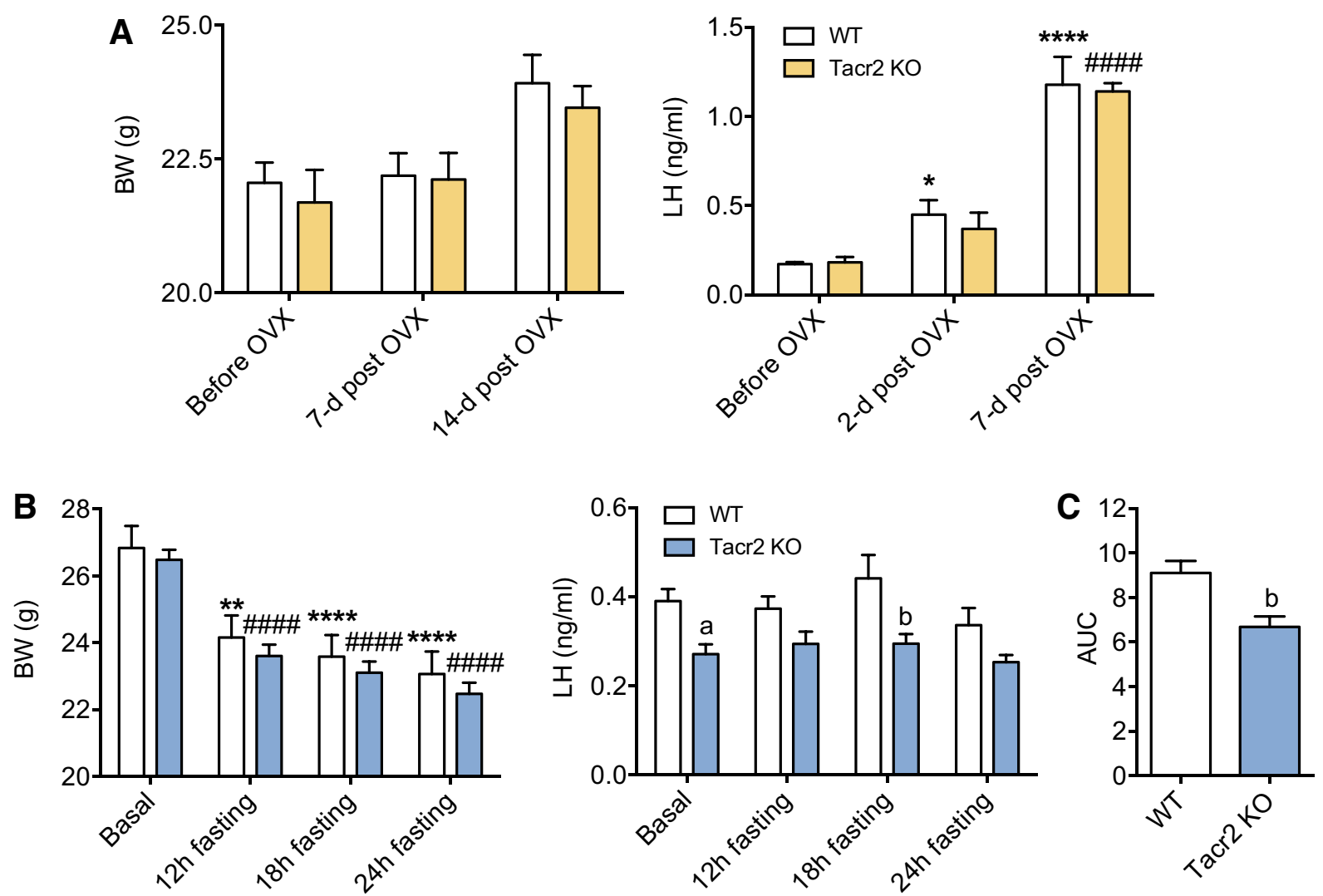

Figure 4. Short-term LH responses to ovariectomized (OVX) and fasting in Tacr $2^{-/-}$mice. A: body weight (BW) changes (at 7 and 14 days post-OVX) and LH responses to ovariectomy (at 2 and 7 days post-OVX) in wild-type (WT) and Tacr $2^{-1-}$ female mice are shown (WT: $n=6$; Tacr2 ${ }^{-1-}: n=7$ ). B: BW changes and LH levels at timed intervals during a 24-h period after food deprivation are presented from Tacr $2^{-1-}$ male mice and their corresponding WT controls (WT: $n=12$; $\operatorname{Tacr}^{-1-}: n=18$ ); the integral (AUC) secretory mass of LH during the 24-h period of fasting in WT and Tacr2 ${ }^{-1-}$ mice is shown in $C$. Data are presented as means $\pm \mathrm{SE}$. Statistical significance was determined by two-way ANOVA followed by Bonferroni's post hoc test: $* P<0.05$ vs. corresponding control values (basal); $* * P<0.001$ vs. corresponding control values (basal); $* * * * \# \# \# \# P<0.0001$ vs. corresponding control values (basal); ${ }^{\mathrm{a}} P<0.05$ vs. corresponding WT values; and ${ }^{\mathrm{b}} P<0.01$ vs. corresponding WT values. AUC, area under the curve.

\section{DISCUSSION}

Our understanding of the central pathways that regulate GnRH neurons remains incomplete. Yet, kisspeptins have been shown to play a major physiological role in the control of GnRH secretion and the function of the reproductive axis, as solidly documented by pharmacological and genetic studies in humans and rodents $(2,5)$. In addition, compelling data have previously shown that not only NKB and its receptor NK3R but also other members of the tachykinin family participate in the control of GnRH neurosecretion and different aspects of reproductive function, seemingly via close interaction with the kisspeptin pathway $(3,7,20,31,32,36$, 48). However, due to the intense cross talk and partial redundancy of the elements of the TAC signaling, as defined by multiple ligands (SP, NKA, and NKB) and receptors (NK1R, NK2R, and NK3R), teasing apart the individual roles of the specific TAC pathways has remained complex and elusive. In this scenario, analysis of human mutations and murine models of inactivation of the genes encoding TAC ligands and receptors has been illustrative, although not fully conclusive, due to species differences, putative compensatory responses, and the divergent characterization of different mouse lines, with genetic inactivation of the various TAC ligands or receptors. In this context, characterization of the physiological roles of NKA/NK2R pathways in the control of reproduction has lagged behind and was mostly based on pharmacological analyses or studies in murine models with congenital ablation of the Tac1 gene, which encodes both SP and NKA. We report herein the first systematic characterization of a novel murine line in which the Tacr 2 gene has been ablated (aka, the $\mathrm{Tacr}^{-/-}$mouse), thus allowing to dissect out the putative roles of NK2R signaling in the control of different aspects of reproductive function, from puberty onset and gonadotropin secretory profiles to sexual behavior. These studies come to cover a persistent gap of knowledge and provide an estimation of the actual physiological relevance of NK2R pathways within the TAC family.

Our data refine and complement recent studies addressing the role of NKA in the control of puberty and reproduction in the mouse (36). In line with previous reports (39), central administration of the agonist of NK2R GR 64349 evoked unambiguous LH responses in control male and female mice, which our current data document are similar in magnitude to those evoked by agonists of other TAC receptors, such as NK1R and NK3R. This pharmacological evidence supports the involvement of NK2R signaling in the stimulatory control of the gonadotropin axis. Intriguingly, however, 

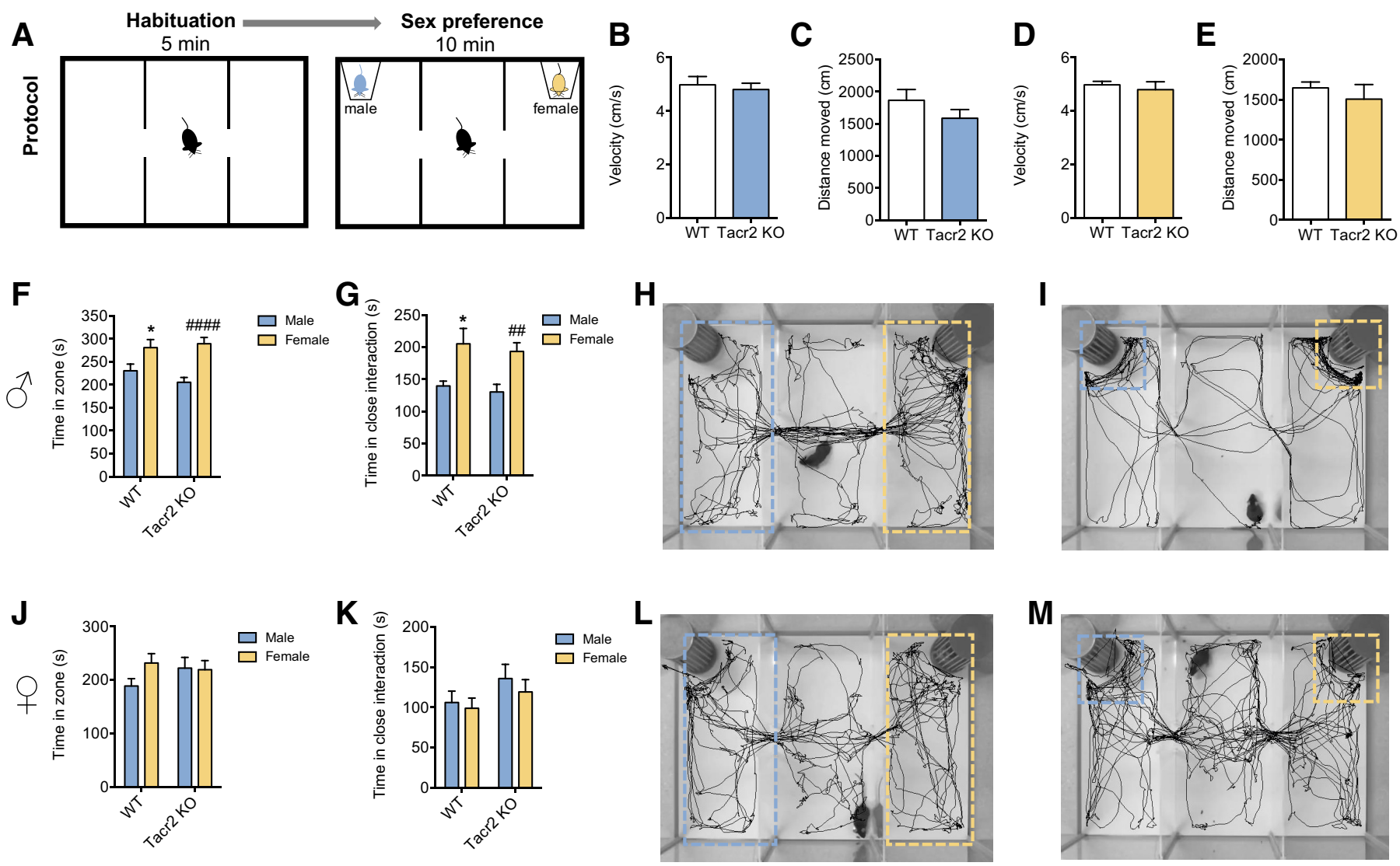

Figure 5. Analysis of social sex preference behavior in $\mathrm{Tacr}^{-/-}$male and female mice. The three-chamber social interaction paradigm testing sex preference was applied. A: representative images illustrating the protocol used are shown. $B$ and $C$ : mean velocity and distance covered, respectively, during the habituation for sex preference tests for wild-type (WT) (white) and Tacr $2^{-/-}$male mice are plotted (WT: $n=8 ;$ Tacr $\left.2^{-/-}: n=15\right)$. In addition, mean velocity $(D)$ and distance covered $(E)$ during the habituation for sex preference tests for WT (white) and Tacr $2^{-1-}$ (blue) female mice are presented (WT: $\left.n=10 ; \operatorname{Tacr}^{-1-}: n=12\right)$. F: data of WT and Tacr2 ${ }^{-1-}$ males when tested for their preference of female over male odor are displayed. G: data of close interaction in similar preference tests are shown. $H$ and $l$ : representative images illustrating the results of sex preference test for Tacr $2^{-1-}$ male mouse are shown. J: in addition, data of WT and Tacr $2^{-1-}$ females when tested for their preference of male over female odor are displayed. $K$ : data of close interaction in similar preference tests are presented. $L$ and $M$ : finally, representative images illustrating the results of sex preference test for Tacr $2^{-1-}$ female mouse are shown. Data are presented as means \pm SE. Statistical significance was determined by Student's $t$ tests: $* P<0.05$ vs. corresponding male time values for WT; \#\#P<0.001 vs. corresponding male time values for Tacr2 $\mathrm{KO}$ and \#\#\# $<0.001$ vs. corresponding male time values for Tacr ${ }^{-/-}$ mice.

despite the proven ablation of Tacr 2 expression in our model, $\mathrm{LH}$ responses to the selective NK2R agonist were only partially blunted in female $\mathrm{TaCr}^{-/-}$mice, while in null males, LH secretion in response to the NKA agonist GR 64349 was only marginally reduced at late timepoints. The possibility that this merely stems from the spurious, nonphysiological activation of other NK receptors by the agonist can be ruled out, since the doses of GR 64349 used were in the low range (7), and this compound has been defined as a bona fide agonist, with much higher selectivity for NK2R over NK1R and NK3R (37). Hence, while some degree of promiscuity at the receptor level of the agonist used may explain the partially preserved LH responses, this is likely to reflect the physiological redundancy of NKA signaling, which might also involve the collateral activation of other TAC receptors. This is further supported by the fact that $\mathrm{LH}$ responses to the NK2R agonist could be totally blocked in $\mathrm{TaCr}^{-/-}$males pretreated with the pharmacological antagonist of NK3R. In the same vein, the phenotypic impact of genetic ablation of Tac1, encoding SP and NKA, has been recently shown to be augmented by the concomitant elimination of Tac2, encoding NKB (30). In any event, it is interesting to note that the impact of Tacr2 elimination on LH secretory responses to the NKA agonist was higher in females, suggesting that the degree of redundancy and compensation among the TAC pathways is lower than in males. This suggests a greater physiological relevance of NK2R signaling in the control of the female (vs. male) gonadotropic axis. In good agreement, the reproductive impact of individual or combined Tac1 and Tac2 ablation was higher in females $(27,30,32)$, suggesting that the female reproductive axis is more sensitive to alterations in TAC signaling. Generation of novel murine models, with combined ablation of Tacr 2 and Tacr1 and/or Tacr3, may help to further expose the degree of redundancy and compensation among the different TAC signaling pathways.

Inactivating mutations of the $\mathrm{NKB} / \mathrm{NK} 3 \mathrm{R}$ systems cause failure of puberty of central origin in humans, whereas mice with genetic inactivation of various elements of the TAC signaling pathways display variable, but milder, alterations, which in general were stronger in females. Yet, congenital ablation of Tacr3 (encoding NK3R) failed to alter puberty 
Figure 6. Analysis of metabolic responses in $\mathrm{TaCr}^{-1-}$ male mice in lean and obesogenic conditions. A: we represent mean $\mathrm{LH}$ levels of wild-type (WT) and $\mathrm{Tacr}^{-/-}$male mice fed with standard, chow diet or high-fat diet (HFD) (chow: WT $n=12$; Tacr2 $^{-/-} n=12$; HFD: WT $n=5$; $\left.\operatorname{Tacr2}^{-/-} n=13\right)$. $B$ and $C$ : body weight (BW) gain and basal glucose levels are presented from WT and Tacr2 ${ }^{-l-}$ mice, fed chow or HFD, at 6 mo of age. $D$ and $E$ : in addition, we show results from glucose tolerance tests (GTTs), represented as 120-min time-course profile after intraperitoneal injection of a glucose bolus, and net increment of integral (AUC) glucose levels during GTT. F and G: finally, similar time-course and integral AUC changes in blood glucose levels during insulin tolerance tests (ITTs) are presented. Data are presented as means + SE. Statistical significance was determined by two-way ANOVA followed by Bonferroni's post hoc test: $* * * / \# \# \# P<0.001$ vs. corresponding control values; \#\#\#\# $<0.0001$ vs. corresponding control values; and ${ }^{\mathrm{a}} P<0.05$ Tacr2 $\mathrm{KO}$ vs. corresponding WT values in HFD.
A

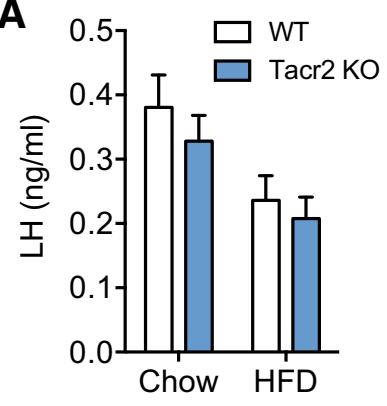

D

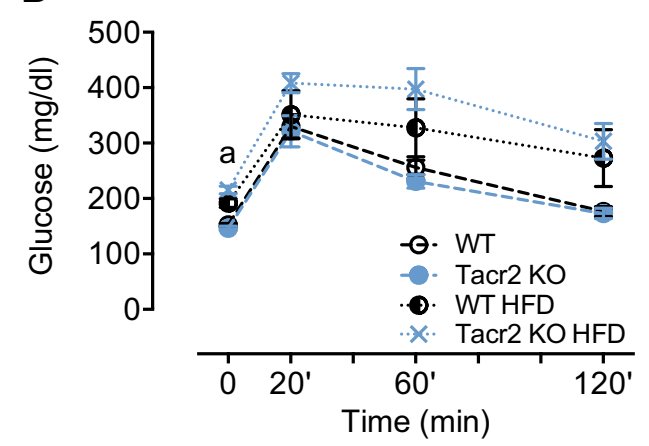

$\mathbf{F}$

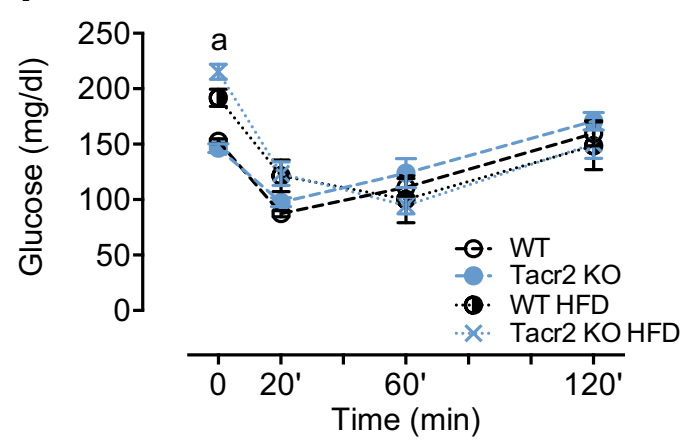

B

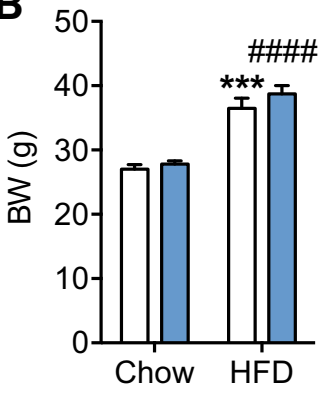

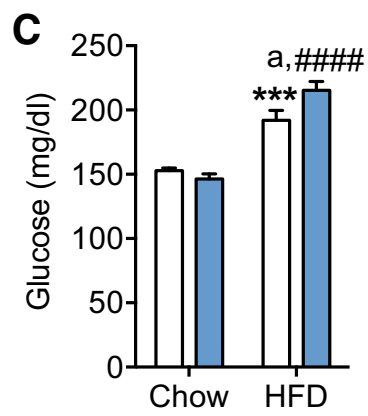
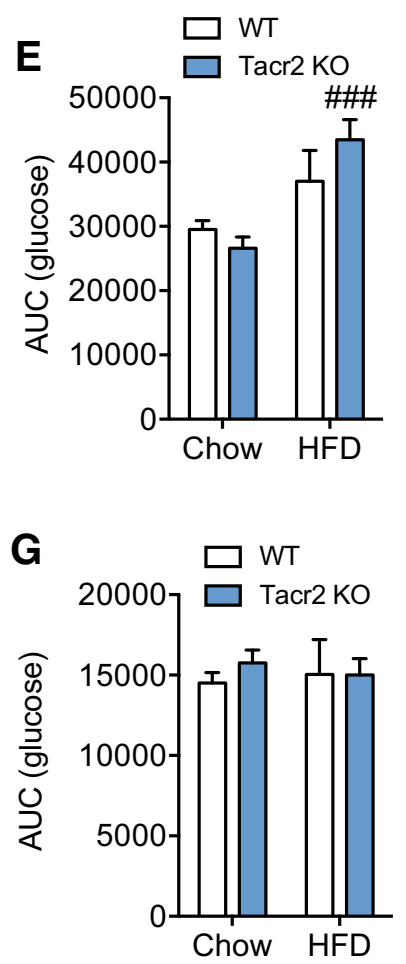

onset in either male or female mice (28), while the pubertal phenotype of Tacr1 null mice has not been reported. Our current data set expands those previous observations and demonstrates normal timing of puberty in $\mathrm{Tacr} 2^{-/-}$mice of either sex. Interestingly, ablation of Tac1 or combined elimination of Tac1/Tac2 did cause overt alterations of puberty in male and female mice (30), suggesting that the joint absence of several TACs is needed to overtly perturb puberty onset in rodents of both sexes. The fact that individual elimination of Tacr 3 or Tacr 2 did not alter pubertal timing in mice further emphasizes the functional redundancy of the TAC system also in terms of pubertal control; a contention that might be confirmed by compound ablation of all TAC receptors in future studies. At a physiological level, these data suggest that the biological effects of the different TACs will depend on the expression level of the three TAC receptors in a given cell target.

Similar redundancy appears to operate in terms of maintenance of fertility, so that both male and female Tacr $2^{-/-}$ mice produced litters when crossed with WT counterparts and displayed grossly normal reproductive indices, such as preserved estrous cyclicity in females. This is in line with the preserved fertility of $\mathrm{TaCr}^{-/-}$mice (34), whereas $\mathrm{Tacr}^{-/-}$ females were subfertile (28). In contrast, individual or compound ablation of the genes encoding the TAC ligands Tac1 and Tac2 resulted in subfertility, with the greatest perturbation being observed in Tac1/Tac2 null mice, which displayed infertility in $80 \%$ of the females (30). Preservation of fertility in mice lacking NK2R signaling was not seemingly attained by developmental compensation in our congenital $\mathrm{KO}$ model, as suggested by the conserved hypothalamic expression of key central elements of the reproductive axis, such as the genes encoding NKB, NK1R and NK3R, Kiss1, dynorphin, and $\mathrm{GnRH}$. In addition, functional $(\mathrm{LH})$ responses to central administration of other activators of the $\mathrm{GnRH} /$ gonadotropin axis, such as agonists of SP, NKB, and kisspeptin, were maintained in $\mathrm{TaCr}^{-/-}$mice; an observation that also argues against a major primary defect at the pituitary level due to congenital ablation of NK2R, in line with previous studies reporting no changes in $\mathrm{LH}$ secretion by rat 
pituitaries from gonadal-intact rats, after challenge with either NKA (35) or an NK2R antagonist in vitro (49). Therefore, considering that pharmacological activation of NK2R can stimulate the gonadotropic axis, it is tenable that the lack of overt reproductive phenotypes in $\mathrm{TaCr}^{-/-}$mice might derive from the cross talk and redundancy among the different TAC pathways, acting likely at central levels, rather than a potential compensation via overexpression of complementary regulatory pathways. Admittedly, however, other compensatory events (e.g., adaptative connectivity) cannot be ruled out on the basis of our current data.

Notwithstanding, while gross fertility was preserved, the fecundity of Tacr 2 null mice, especially of females, appeared to be modestly compromised, as suggested by their longer breeding intervals than controls. In the same line, Tacr $2^{-/-}$ female mice displayed altered patterns of LH pulsatility, as defined by lower mean and basal LH levels than corresponding control females, although the number of LH pulses over the 3-h period of sampling was preserved. These data point out that, despite the potential redundancy among the TAC pathways, NK2R plays a genuine, specific role in defining specific aspects of LH pulsatility. Of note, previous evidence had demonstrated that, although the stimulatory effects of NKA on GnRH/LH secretion require a preserved kisspeptin signaling (36), Tacr2 is not expressed in Kiss1 (or GnRH) neurons, suggesting a primary site of action of NKA on upstream afferents to Kiss1 neurons (15). These might include Tac1 neurons in the ventromedial hypothalamus (VMH), which express Tacr2, as well as unidentified neurons from the ARC and $\mathrm{VMH}(36)$. This is in contrast with the proposed network for the stimulatory effects of NKB, which would take place directly on ARC KNDy neurons, by auto- or parasynaptic loops. Interestingly, this KNDy neuronal population has been proposed as an essential component of the GnRH pulse generator, which likely dictates not only the pulse interval but also other features of the secretory profile of GnRH. On the basis of our data, the NKA/NK2R pathway appears to play a relevant role in defining the basal interpulse levels of GnRH/LH secretion. Admittedly, other neurotransmitters with proven roles in the central control of GnRH neurosecretion (50) may contribute also in conveying at least part of NKA/NK2R effects on LH secretion.

Subtle alterations were also noticed when analyzing single-point LH levels in $\mathrm{Tacr}^{-/-}$mice subjected to manipulations known to perturb the normal functioning of the gonadotropic axis. On one hand, short-term responses to ovariectomy appeared to be marginally blunted in $\mathrm{Tacr}^{-/-}$ female mice, as LH levels at 2 days after OVX were not significantly increased over basal levels, in contrast with the significant rise detected in control animals. On the other hand, the secretory mass of LH over a 24 -h period of fasting, a manipulation known to suppress LH secretion, was significantly lower in $\mathrm{Tacr}^{-/}$- male mice than in their corresponding controls. A tenable explanation for both findings is that the ablation of a stimulatory pathway, such as NK2R, would result in a partially defective activation of the GnRH/LH system immediately after lifting the negative feedback of sex steroids by OVX but a greater suppression of LH secretion in conditions driving an inhibitory input to the reproductive axis, such as fasting. Admittedly, however, the net differences caused by Tacr 2 ablation were rather modest and, in fact, the absence of NK2R signaling did not prevent (but rather modestly attenuated) LH secretory patterns. These observations further emphasize the detectable, but modest, physiological role of this TAC pathway in the control of the gonadotropic axis.

In addition to neuroendocrine responses, basic aspects of social and sexual behavior were explored in $\mathrm{Tacr}^{-1-}$ mice. These analyses were fueled by previous studies in mice congenitally deficient for Tac1 or Tacr1 which evidenced that the SP/NK1R pathway plays a role in emotional behavior (51, 52). In addition, previous reports had documented that ablation of Tacr1, but not of Tac1, results in diminished attraction by female pheromones and blunted sexual behavior (34). In clear contrast, our current analyses failed to detect any defects in the exploration of female urine by $\mathrm{TaCr}^{-/-}$ male mice. Similarly, social sex preference was not affected by congenital ablation of Tacr 2 , even in male mice, in which the stereotyped preference for the female was fully preserved in Tacr2 null animals. Albeit more incisive sex behavioral tests were not applied (e.g., assessing lordosis or copulatory efficiency) due to the lack of behavioral phenotypes in our initial analyses, our current data strongly suggest that, contrary to Tacr1, Tacr2 pathways are dispensable for the manifestation of basic adult social and sex preference behavior in mice.

Similar to kisspeptins, for which distinct metabolic roles have been recently proposed $(53,54)$, previous evidence strongly suggested that TAC pathways putatively participate in the control of key aspects of metabolism, such as body weight and composition, feeding behavior, and glucose homeostasis. In detail, initial studies using Tacr1 KO mice revealed a potential metabolic role of NK1R signaling in metabolic responses to obesogenic insults, as Tacr1-deficient animals showed reduced body weight gain and circulating levels of leptin and insulin, as well as improved glucose homeostasis in response to an HFD (55). In good agreement, later analyses in Tac1 null mice have documented a role of SP signaling in metabolic responses to obesity, so that congenital ablation of Tac1 conferred resistance to obesity and improved glucose tolerance and insulin sensitivity under an HFD $(56,57)$. Of note, ablation of Tac1 might positively impact metabolic profiles by elimination of SP and/or NKA, the actions of the latter being mediated by NK2R. However, our current data argue against this possibility, since NK2Rdeficient mice, under normal diet, displayed similar body weight gain and composition as their paired fed littermates, neither did they show alterations in energy expenditure, respiratory quotient, and food intake. Moreover, while exposure to an HFD caused an increase in body weight that was similar in control and $\mathrm{TaCr}^{-/-}$male mice, basal glucose levels were higher in Tacr2 null animals fed a HFD, which also displayed worse indices of glucose intolerance, without changes in insulin sensitivity. Altogether, this evidence suggests that, if any, the role of NKA/NK2R signaling in the control of metabolism is opposite to that of SP/NK1R, both being seemingly engaged in a reciprocal manner in the modulation of glucose homeostasis, especially in obesogenic conditions. As a final note, despite previous pharmacological evidence suggesting a putative role of NKA in the control of cardiovascular function (58), our current analyses failed to detect any alteration in blood pressure in our Tacr2 ${ }^{-/-}$mice, which 

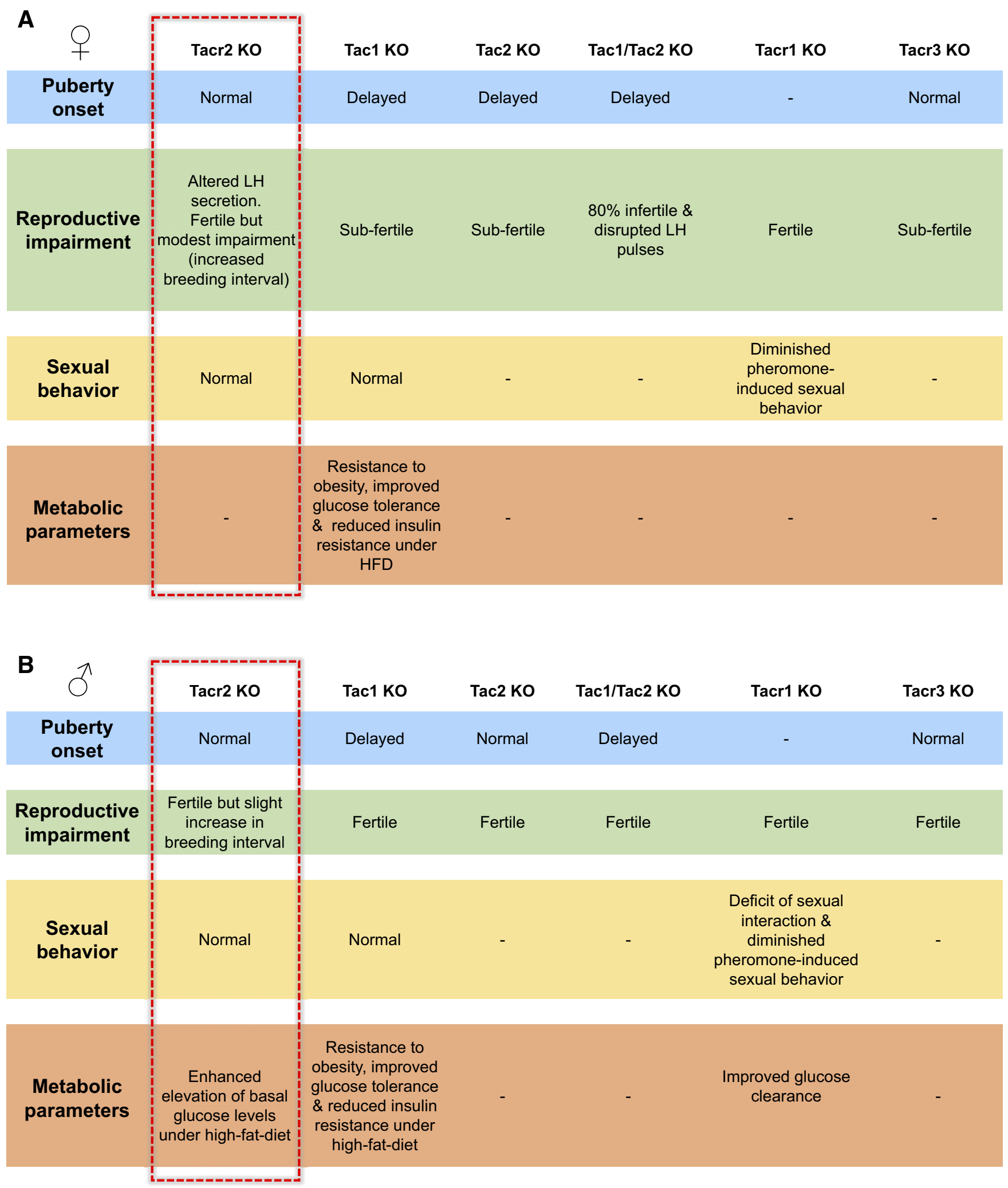

Figure 7. Summary of major phenotypic features of $\mathrm{Tacr}^{-1-}$ mouse and comparison with other genetic models of altered tachykinin (TAC) signaling. The major phenotypic features of female $(A)$ and male $(B) T a c r 2^{-1-}$ mouse, as compared with those described previously for other genetically modified mouse lines, with congenital ablation of the genes encoding TACs (Tac1 and Tac2) or other TAC receptors (Tacr1 and Tacr3), are synoptically presented. 
were normotensive in both sexes. These findings are in line with previous pharmacological studies showing that blockade of NK2R did not cause changes in blood pressure or heart rate in conscious rats (59). Deeper analyses in our genetic model are deemed to assess whether other parameters of cardiovascular function might be altered in the congenital absence of NK2R.

In conclusion, our present study is the first to document that global disruption of the Tacr2 gene, which results in ablation of NK2R, leads to detectable, albeit discrete, deficits in the functioning of the reproductive axis, including the suppression of basal and mean LH levels and trend for lengthening of breeding intervals but without preventing fertility or basic social sex behavior. Of note, pharmacological tests in our model of congenital ablation of Tacr2 allowed to surface sex differences in the degree of redundancy between this and other TAC pathways. In addition, our data also suggest that the NK2R pathway might contribute to keeping glucose homeostasis at check in obesogenic conditions, playing a role opposite to NK1R. All in all, our current data set adds to previous knowledge on the reproductive (as well as behavioral and metabolic) roles of TAC pathways (see Fig. 7) and helps to weight the actual physiological relevance of NK2R signaling in mediating TAC actions in these neuroendocrine axes.

\section{ACKNOWLEDGMENTS}

The Tacr2 $^{-/}$mouse line was made available by Menarini Ricerche S.p.A. (Italy) for basic research purposes; the authors thank to Dr. Stephania Meini for support in providing this mouse line.

\section{GRANTS}

This work was supported by Grant BFU2017-83934-P (Ministerio de Economía y Competitividad, Spain; co-funded with EU funds from FEDER Program), Project PIE-00005 (Instituto de Salud Carlos III, Ministerio de Sanidad, Spain), Project P12-FQM-01943 (Junta de Andalucía, Spain), and Project REP-655232 (ReprObesity; European Union). CIBER Fisiopatología de la Obesidad y Nutrición is an initiative of Instituto de Salud Carlos III, Spain.

\section{DISCLOSURES}

No conflicts of interest, financial or otherwise, are declared by the authors.

\section{AUTHOR CONTRIBUTIONS}

A.R-R., M.T-S., and M.L.C. conceived and designed research; E.T., I.V., D.F., V.H., F.G., and R.P. performed experiments; E.T., A.R-R., M.T-S., I.V., D.F., V.H., F.G., S.L., V.N., R.P., and M.L.C. analyzed data; E.T., A.R-R., M.T-S., S.L., V.M.N., R.P., and M.L.C. interpreted results of experiments; E.T. and I.V. prepared figures; M.T-S. drafted manuscript; E.T., A.R-R., M.T-S., S.L., V.N., and M.L.C. edited and revised manuscript; E.T., A.R-R., M.T-S., I.V., D.F., V.H., F.G., S.L., V.N., R.P., and M.L.C. approved final version of manuscript.

\section{REFERENCES}

1. Herbison AE. Control of puberty onset and fertility by gonadotropinreleasing hormone neurons. Nat Rev Endocrinol 12: 452-466, 2016. doi:10.1038/nrendo.2016.70.
2. Pinilla L, Aguilar E, Dieguez C, Millar RP, Tena-Sempere M. Kisspeptins and reproduction: physiological roles and regulatory mechanisms. Physiol Rev 92: 1235-1316, 2012. doi:10.1152/ physrev.00037.2010.

3. Garcia-Galiano D, van Ingen Schenau D, Leon S, Krajnc-Franken MA, Manfredi-Lozano M, Romero-Ruiz A, Navarro VM, Gaytan F, van Noort PI, Pinilla L, Blomenrohr M, Tena-Sempere M. Kisspeptin signaling is indispensable for neurokinin B, but not glutamate, stimulation of gonadotropin secretion in mice. Endocrinology 153: 316328, 2012. doi:10.1210/en.2011-1260.

4. Hellier V, Brock O, Candlish M, Desroziers E, Aoki M, Mayer C, Piet R, Herbison A, Colledge WH, Prevot V, Boehm U, Bakker J. Female sexual behavior in mice is controlled by kisspeptin neurons. Nat Commun 9: 400, 2018. doi:10.1038/s41467-017-02797-2.

5. Terasawa E, Guerriero KA, Plant TM. Kisspeptin and puberty in mammals. Adv Exp Med Biol 784: 253-273, 2013. doi:10.1007/978-14614-6199-9_12.

6. Goodman RL, Lehman MN, Smith JT, Coolen LM, de Oliveira CV, Jafarzadehshirazi MR, Pereira A, Iqbal J, Caraty A, Ciofi P, Clarke IJ. Kisspeptin neurons in the arcuate nucleus of the ewe express both dynorphin A and neurokinin B. Endocrinology 148: 5752-5760, 2007. doi:10.1210/en.2007-0961.

7. Navarro VM, Bosch MA, Leon S, Simavli S, True C, Pinilla L, Carroll RS, Seminara SB, Tena-Sempere M, Ronnekleiv OK, Kaiser UB. The integrated hypothalamic tachykinin-kisspeptin system as a central coordinator for reproduction. Endocrinology 156: 627-637, 2015. doi:10.1210/en.2014-1651.

8. Noritake K, Matsuoka T, Ohsawa T, Shimomura K, Sanbuissho A Uenoyama Y, Maeda K, Tsukamura H. Involvement of neurokinin receptors in the control of pulsatile luteinizing hormone secretion in rats. J Reprod Dev 57: 409-415, 2011. doi:10.1262/jrd.11-002S.

9. Ruiz-Pino F, Navarro VM, Bentsen AH, Garcia-Galiano D, SanchezGarrido MA, Ciofi P, Steiner RA, Mikkelsen JD, Pinilla L, TenaSempere $\mathbf{M}$. Neurokinin B and the control of the gonadotropic axis in the rat: developmental changes, sexual dimorphism, and regulation by gonadal steroids. Endocrinology 153: 4818-4829, 2012. doi:10.1210/en.2012-1287.

10. Pennefather JN, Lecci A, Candenas ML, Patak E, Pinto FM, Maggi CA. Tachykinins and tachykinin receptors: a growing family. Life Sci 74: 1445-1463, 2004. doi:10.1016/j.Ifs.2003.09.039.

11. Steinhoff MS, von Mentzer B, Geppetti P, Pothoulakis C, Bunnett NW. Tachykinins and their receptors: contributions to physiological control and the mechanisms of disease. Physiol Rev 94: 265-301, 2014. doi:10.1152/physrev.00031.2013.

12. Satake H, Aoyama M, Sekiguchi T, Kawada T. Insight into molecular and functional diversity of tachykinins and their receptors. Protein Pept Lett 20: 615-627, 2013. doi:10.2174/0929866511320060002.

13. Maggi CA. The mammalian tachykinin receptors. Gen Pharmacol 26: 911-944, 1995. doi:10.1016/0306-3623(94)00292-U.

14. de Croft S, Boehm U, Herbison AE. Neurokinin B activates arcuate kisspeptin neurons through multiple tachykinin receptors in the male mouse. Endocrinology 154: 2750-2760, 2013. doi:10.1210/ en.2013-1231.

15. Leon S, Navarro VM. Novel biology of tachykinins in gonadotropinreleasing hormone secretion. Semin Reprod Med 37: 109-118, 2019. doi:10.1055/s-0039-3400252.

16. Topaloglu AK, Reimann F, Guclu M, Yalin AS, Kotan LD, Porter KM, Serin A, Mungan NO, Cook JR, Ozbek MN, Imamoglu S, Akalin NS, Yuksel B, O'Rahilly S, Semple RK. TAC3 and TACR3 mutations in familial hypogonadotropic hypogonadism reveal a key role for Neurokinin B in the central control of reproduction. Nat Genet 41: 354-358, 2009. doi:10.1038/ng.306.

17. Young J, Bouligand J, Francou B, Raffin-Sanson ML, Gaillez S Jeanpierre M, Grynberg M, Kamenicky P, Chanson P, BraillyTabard S, Guiochon-Mantel A. TAC3 and TACR3 defects cause hypothalamic congenital hypogonadotropic hypogonadism in humans. J Clin Endocrinol Metab 95: 2287-2295, 2010. doi:10. 1210/jc.2009-2600.

18. Cheng G, Coolen LM, Padmanabhan V, Goodman RL, Lehman MN. The kisspeptin/neurokinin B/dynorphin (KNDy) cell population of the arcuate nucleus: sex differences and effects of prenatal testosterone in sheep. Endocrinology 151: 301-311, 2010. doi:10.1210/ en.2009-0541. 
19. Hrabovszky E, Sipos MT, Molnar CS, Ciofi P, Borsay BA, Gergely P, Herczeg L, Bloom SR, Ghatei MA, Dhillo WS, Liposits Z. Low degree of overlap between kisspeptin, neurokinin B, and dynorphin immunoreactivities in the infundibular nucleus of young male human subjects challenges the KNDy neuron concept. Endocrinology 153: 4978-4989, 2012. doi:10.1210/en.2012-1545.

20. Navarro VM, Gottsch ML, Chavkin C, Okamura H, Clifton DK, Steiner RA. Regulation of gonadotropin-releasing hormone secretion by kisspeptin/dynorphin/neurokinin B neurons in the arcuate nucleus of the mouse. J Neurosci 29: 11859-11866, 2009. doi:10.1523/ JNEUROSCI.1569-09.2009.

21. Ramaswamy S, Seminara SB, Ali B, Ciofi P, Amin NA, Plant TM. Neurokinin B stimulates GnRH release in the male monkey (Macaca mulatta) and is colocalized with kisspeptin in the arcuate nucleus. Endocrinology 151: 4494-4503, 2010. doi:10.1210/en.2010-0223.

22. Lehman MN, Coolen LM, Goodman RL. Minireview: kisspeptin/neurokinin B/dynorphin (KNDy) cells of the arcuate nucleus: a central node in the control of gonadotropin-releasing hormone secretion. Endocrinology 151: 3479-3489, 2010. doi:10.1210/en.2010-0022.

23. Navarro VM, Tena-Sempere M. Neuroendocrine control by kisspeptins: role in metabolic regulation of fertility. Nat Rev Endocrinol 8: 40-53, 2011. doi:10.1038/nrendo.2011.147.

24. Clarkson J, Han SY, Piet R, McLennan T, Kane GM, Ng J, Porteous RW, Kim JS, Colledge WH, Iremonger KJ, Herbison AE. Definition of the hypothalamic GnRH pulse generator in mice. Proc Natl Acad Sci USA 114: E10216-E10223, 2017. doi:10.1073/pnas.1713897114.

25. Voliotis M, Li XF, De Burgh R, Lass G, Lightman SL, O'Byrne KT, Tsaneva-Atanasova $\mathbf{K}$. The origin of $\mathrm{GnRH}$ pulse generation: an integrative mathematical-experimental approach. J Neurosci 39: 9738-9747, 2019. doi:10.1523/JNEUROSCI.0828-19.2019.

26. Grachev P, Li XF, Lin YS, Hu MH, Elsamani L, Paterson SJ, Millar RP, Lightman SL, O'Byrne KT. GPR54-dependent stimulation of luteinizing hormone secretion by neurokinin $\mathrm{B}$ in prepubertal rats. PLoS One 7: e44344, 2012. doi:10.1371/journal.pone.0044344.

27. True C, Nasrin Alam S, Cox K, Chan YM, Seminara SB. Neurokinin $B$ is critical for normal timing of sexual maturation but dispensable for adult reproductive function in female mice. Endocrinology 156: 1386-1397, 2015. doi:10.1210/en.2014-1862.

28. Yang JJ, Caligioni CS, Chan YM, Seminara SB. Uncovering novel reproductive defects in neurokinin $B$ receptor null mice: closing the gap between mice and men. Endocrinology 153: 1498-1508, 2012. doi:10.1210/en.2011-1949.

29. Gianetti E, Tusset C, Noel SD, Au MG, Dwyer AA, Hughes VA, Abreu AP, Carroll J, Trarbach E, Silveira LF, Costa EM, de Mendonca BB, de Castro M, Lofrano A, Hall JE, Bolu E, Ozata M, Quinton R, Amory JK, Stewart SE, Arlt W, Cole TR, Crowley WF, Kaiser UB, Latronico AC, Seminara SB. TAC3/TACR3 mutations reveal preferential activation of gonadotropin-releasing hormone release by neurokinin $B$ in neonatal life followed by reversal in adulthood. J Clin Endocrinol Metab 95: 2857-2867, 2010. doi:10.1210/ jc.2009-2320.

30. Leon S, Fergani C, Talbi R, Maguire CA, Gerutshang A, Seminara SB, Navarro VM. Tachykinin signaling is required for the induction of the preovulatory LH surge and normal LH pulses. Neuroendocrinology In press. doi:10.1159/000509222.

31. Ruiz-Pino F, Garcia-Galiano D, Manfredi-Lozano M, Leon S, Sanchez-Garrido MA, Roa J, Pinilla L, Navarro VM, Tena-Sempere M. Effects and interactions of tachykinins and dynorphin on FSH and LH secretion in developing and adult rats. Endocrinology 156: 576588, 2015. doi:10.1210/en.2014-1026.

32. Simavli S, Thompson IR, Maguire CA, Gill JC, Carroll RS, Wolfe A, Kaiser UB, Navarro VM. Substance $p$ regulates puberty onset and fertility in the female mouse. Endocrinology 156: 2313-2322, 2015. doi:10.1210/en.2014-2012.

33. Dornan WA, Malsbury CW, Penney RB. Facilitation of lordosis by injection of substance $P$ into the midbrain central gray. Neuroendocrinology 45: 498-506, 1987. doi:10.1159/000124781.

34. Berger A, Tran AH, Dida J, Minkin S, Gerard NP, Yeomans J, Paige CJ. Diminished pheromone-induced sexual behavior in neurokinin-1 receptor deficient (TACR1(-/-)) mice. Genes Brain Behav 11: 568-576, 2012. doi:10.1111/j.1601-183X.2012.00787.x.

35. Kalra PS, Sahu A, Bonavera JJ, Kalra SP. Diverse effects of tachykinins on luteinizing hormone release in male rats: mechanism of action. Endocrinology 131: 1195-1201, 1992. doi:10.1210/endo.131.3.1380435.

36. Leon S, Fergani C, Talbi R, Simavli S, Maguire CA, Gerutshang A, Navarro VM. Characterization of the role of NKA in the control of puberty onset and gonadotropin release in the female mouse. Endocrinology 160: 2453-2463, 2019. doi:10.1210/en.2019-00195.

37. Deal MJ, Hagan RM, Ireland SJ, Jordan CC, McElroy AB, Porter B, Ross BC, Stephens-Smith M, Ward P. Conformationally constrained tachykinin analogues: potent and highly selective neurokinin NK-2 receptor agonists. J Med Chem 35: 4195-4204, 1992. doi:10.1021/ jm00100a027.

38. Marson L, Thor KB, Katofiasc M, Burgard EC, Rupniak NMJ. Prokinetic effects of neurokinin-2 receptor agonists on the bladder and rectum of rats with acute spinal cord transection. Eur J Pharmacol 819: 261-269, 2018. doi:10.1016/j.ejphar.2017.12.017.

39. Leon S, Barroso A, Vazquez MJ, Garcia-Galiano D, ManfrediLozano M, Ruiz-Pino F, Heras V, Romero-Ruiz A, Roa J, Schutz G, Kirilov M, Gaytan F, Pinilla L, Tena-Sempere M. Direct actions of kisspeptins on $\mathrm{GnRH}$ neurons permit attainment of fertility but are insufficient to fully preserve gonadotropic axis activity. Sci Rep 6: 19206, 2016. doi:10.1038/srep19206.

40. Leon S, Velasco I, Vazquez MJ, Barroso A, Beiroa D, Heras V, RuizPino F, Manfredi-Lozano M, Romero-Ruiz A, Sanchez-Garrido MA, Dieguez C, Pinilla L, Roa J, Nogueiras R, Tena-Sempere M. Sex-biased physiological roles of NPFF1R, the canonical receptor of RFRP3 , in food intake and metabolic homeostasis revealed by its congenital ablation in mice. Metabolism 87: 87-97, 2018. doi:10.1016/j. metabol.2018.07.003.

41. Navarro VM, Ruiz-Pino F, Sanchez-Garrido MA, Garcia-Galiano D, Hobbs SJ, Manfredi-Lozano M, Leon S, Sangiao-Alvarellos S, Castellano JM, Clifton DK, Pinilla L, Steiner RA, Tena-Sempere M. Role of neurokinin $B$ in the control of female puberty and its modulation by metabolic status. J Neurosci 32: 2388-2397, 2012. doi:10.1523/JNEUROSCI.4288-11.2012.

42. Steyn FJ, Wan Y, Clarkson J, Veldhuis JD, Herbison AE, Chen C. Development of a methodology for and assessment of pulsatile luteinizing hormone secretion in juvenile and adult male mice. Endocrinology 154: 4939-4945, 2013. doi:10.1210/en.2013-1502.

43. Roa J, Garcia-Galiano D, Varela L, Sanchez-Garrido MA, Pineda R, Castellano JM, Ruiz-Pino F, Romero M, Aguilar E, Lopez M, Gaytan F, Dieguez C, Pinilla L, Tena-Sempere M. The mammalian target of rapamycin as novel central regulator of puberty onset via modulation of hypothalamic Kiss1 system. Endocrinology 150: 5016-5026, 2009. doi:10.1210/en.2009-0096.

44. Esparza LA, Schafer D, Ho BS, Thackray VG, Kauffman AS. Hyperactive LH pulses and elevated kisspeptin and NKB gene expression in the arcuate nucleus of a PCOS mouse model. Endocrinology 161: bqaa018, 2020. doi:10.1210/endocr/bqaa018.

45. Bilkei-Gorzo A, Racz I, Michel K, Zimmer A. Diminished anxietyand depression-related behaviors in mice with selective deletion of the Tac1 gene. J Neurosci 22: 10046-10052, 2002. doi:10.1523/ JNEUROSCI.22-22-10046.2002.

46. Malkesman O, Scattoni ML, Paredes D, Tragon T, Pearson B, Shaltiel G, Chen G, Crawley JN, Manji HK. The female urine sniffing test: a novel approach for assessing reward-seeking behavior in rodents. Biol Psychiatry 67: 864-871, 2010. doi:10.1016/j. biopsych.2009.10.018.

47. Castellano JM, Navarro VM, Fernandez-Fernandez R, Nogueiras R, Tovar S, Roa J, Vazquez MJ, Vigo E, Casanueva FF, Aguilar E, Pinilla L, Dieguez C, Tena-Sempere M. Changes in hypothalamic KiSS-1 system and restoration of pubertal activation of the reproductive axis by kisspeptin in undernutrition. Endocrinology 146: 39173925, 2005. doi:10.1210/en.2005-0337.

48. Maguire CA, Song YB, Wu M, Leon S, Carroll RS, Alreja M, Kaiser UB, Navarro VM. Tac1 signaling is required for sexual maturation and responsiveness of $\mathrm{GnRH}$ neurons to kisspeptin in the male mouse. Endocrinology 158: 2319-2329, 2017. doi:10.1210/en.2016-1807.

49. Debeljuk L, Bandera R, Bartke A. Effect of a non-peptide NK-2 tachykinin receptor antagonist on $\mathrm{LH}, \mathrm{FSH}$, and prolactin release by rat hemipituitaries in vitro. J Physiol Pharmacol 48: 461-478, 1997.

50. Avendano MS, Vazquez MJ, Tena-Sempere M. Disentangling puberty: novel neuroendocrine pathways and mechanisms for the 
control of mammalian puberty. Hum Reprod Update 23: 737-763, 2017. doi:10.1093/humupd/dm×025.

51. Cao YQ, Mantyh PW, Carlson EJ, Gillespie AM, Epstein CJ, Basbaum Al. Primary afferent tachykinins are required to experience moderate to intense pain. Nature 392: 390-394, 1998. doi:10.1038/32897

52. Santarelli L, Gobbi G, Debs PC, Sibille ET, Blier P, Hen R, Heath MJ. Genetic and pharmacological disruption of neurokinin 1 receptor function decreases anxiety-related behaviors and increases serotonergic function. Proc Natl Acad Sci USA 98: 1912-1917, 2001. doi:10.1073/pnas.98.4.1912.

53. Velasco I, Leon S, Barroso A, Ruiz-Pino F, Heras V, Torres E, Leon M, Ruohonen ST, Garcia-Galiano D, Romero-Ruiz A, SanchezGarrido MA, Olhsson C, Castellano JM, Roa J, Poutanen M, Pinilla L, Vazquez MJ, Tena-Sempere M. Gonadal hormone-dependent vs. -independent effects of kisspeptin signaling in the control of body weight and metabolic homeostasis. Metabolism 98: 84-94, 2019. doi:10.1016/j.metabol.2019.06.007.

54. Wolfe A, Hussain MA. The emerging role(s) for kisspeptin in metabolism in mammals. Front Endocrinol (Lausanne) 9: 184, 2018. doi:10.3389/fendo.2018.00184

55. Karagiannides I, Stavrakis D, Bakirtzi K, Kokkotou E, Pirtskhalava T, Nayeb-Hashemi H, Bowe C, Bugni JM, Nuno M, Lu B, Gerard
NP, Leeman SE, Kirkland JL, Pothoulakis C. Substance P (SP)-neurokinin-1 receptor (NK-1R) alters adipose tissue responses to high-fat diet and insulin action. Endocrinology 152: 2197-2205, 2011. doi:10.1210/en.2010-1345.

56. Karagiannides I, Bakirtzi K, Kokkotou E, Stavrakis D, Margolis KG, Thomou T, Giorgadze N, Kirkland JL, Pothoulakis C. Role of substance $P$ in the regulation of glucose metabolism via insulin signaling-associated pathways. Endocrinology 152: 4571-4580, 2011. doi:10.1210/en.2011-1170.

57. Maguire CA, Leon S, Carroll RS, Kaiser UB, Navarro VM. Altered circadian feeding behavior and improvement of metabolic syndrome in obese Tac1-deficient mice. Int J Obes (Lond) 41: 1798-1804, 2017. doi:10.1038/ijo.2017.185.

58. Kaczyńska K, Jampolska M, Szereda-Przestaszewska M. The role of vagal pathway and NK1 and NK2 receptors in cardiovascular and respiratory effects of neurokinin A. Clin Exp Pharmacol Physiol 43: 818-824, 2016. doi:10.1111/1440-1681.12594.

59. Giuliani S, Guelfi M, Toulouse M, Bueno L, Lecci A, Tramontana M, Criscuoli M, Maggi CA. Effect of a tachykinin NK(2) receptor antagonist, nepadutant, on cardiovascular and gastrointestinal function in rats and dogs. Eur J Pharmacol 415: 61-71, 2001. doi:10.1016/S00142999(01)00794-4. 